\title{
The modularity of the Barth-Nieto quintic and its relatives
}

\author{
K. Hulek, J. Spandaw, B. van Geemen and D. van Straten \\ (Communicated by K. Strambach and S. Weintraub)
}

\begin{abstract}
The moduli space of (1,3)-polarized abelian surfaces with full level-2 structure is birational to a double cover of the Barth-Nieto quintic. Barth and Nieto have shown that these varieties have Calabi-Yau models $Z$ and $Y$, respectively. In this paper we apply the Weil conjectures to show that $Y$ and $Z$ are rigid and we prove that the $L$-function of their common third étale cohomology group is modular, as predicted by a conjecture of Fontaine and Mazur. The corresponding modular form is the unique normalized cusp form of weight 4 for the group $\Gamma_{1}(6)$. By Tate's conjecture, this should imply that $Y$, the fibred square of the universal elliptic curve $S_{1}(6)$, and Verrill's rigid Calabi-Yau $\mathscr{Z}_{A_{3}}$, which all have the same $L$-function, are in correspondence over $\mathbb{Q}$. We show that this is indeed the case by giving explicit maps.
\end{abstract}

\section{Introduction}

The Barth-Nieto quintic is the variety given by the equations

$$
N=\left\{\sum_{i=0}^{5} x_{i}=\sum_{i=0}^{5} \frac{1}{x_{i}}=0\right\} \subset \mathbb{P}_{5}
$$

This singular quintic threefold in $\mathbb{P}_{4}=\left\{\sum_{i=0}^{5} x_{i}=0\right\}$ was studied by Barth and Nieto in [1]. They show that it parametrizes Heisenberg $H_{2,2}$-invariant quartics in $\mathbb{P}_{3}$ which contain a line. A smooth such quartic then contains 32 lines which form two $\mathrm{H}_{2,2}$ orbits of 16 disjoint lines each. Taking the double cover branched along these orbits of lines gives two abelian surfaces which are dual and which have a polarization of type $(1,3)$. This defines a map $\mathscr{A}_{1,3}(2) \rightarrow N$ of degree 2 , where $\mathscr{A}_{1,3}(2)$ is the moduli space of abelian surfaces with (1,3)-polarization and a full level-2 structure. The moduli space $\mathscr{A}_{1,3}(2)$ is birationally equivalent to the inverse image $\tilde{N}$ of $N$ under the the double cover of $\mathbb{P}_{5}$ branched along the union of the 6 hyperplanes $\left\{x_{k}=0\right\}$. (All tildes " $\sim$ ", in this paper denote double covers; resolutions of singularities are denoted by a hat "^,".)

The varieties $N$ and $\tilde{N}$ have smooth (strict) Calabi-Yau models, denoted by $Y$ and $Z$ respectively. Thus they have trivial canonical bundle and $h^{i}(\mathcal{O})=0$ for $0<i<3$. The Euler numbers are $e(Y)=100$ and $e(Z)=80$. The paper [10] gives a different 
proof for the existence of a Calabi-Yau model of $\tilde{N}$, using a Siegel modular form and the birationality of $\mathscr{A}_{1,3}(2)$ and $\tilde{N}$.

In this paper we prove that $Y$ and $Z$ are rigid. In fact, this determines all Hodge numbers and we obtain the following result.

Theorem 2.1. Both $Y$ and $Z$ have Hodge numbers $h^{p, q}=0$ except the following:

$$
\begin{gathered}
h^{0,0}=h^{3,0}=h^{0,3}=h^{3,3}=1, \\
h^{1,1}(Y)=h^{2,2}(Y)=50, \quad h^{1,1}(Z)=h^{2,2}(Z)=40 .
\end{gathered}
$$

In particular, both manifolds are rigid.

The most difficult part of the proof of Theorem 2.1 is the determination of $h^{2,2}$ and $h^{2,1}$. We exploit the fact that $Y$ is defined over $\mathbb{Z}$ and that we can replace $Z$ by another model, $\tilde{Y}$, which is defined over $\mathbb{Z}$ and which has $H^{3}(\tilde{Y}) \cong H^{3}(Z)$. Following the method pioneered in [19], we reduce modulo some primes where $Y$ and $\tilde{Y}$ have good reduction and explicitly count the number of points on the reductions with the help of a computer. Using the Lefschetz fixed point formula and the Riemann hypothesis for varieties over finite fields leads to the desired result.

We summarize the maps between the various varieties by the commutative diagram

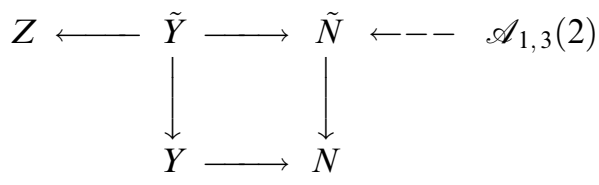

where the horizontal and vertical maps are generically $1: 1$ and $2: 1$, respectively. As mentioned above, we usually work with $\tilde{Y}$ instead of $Z$, even though it is not CalabiYau, since it is defined over $\mathbb{Z}$.

According to Theorem 2.1 the étale cohomology groups $H_{\mathrm{ett}}^{3}(Y)$ and $H_{\mathrm{ett}}^{3}(\tilde{Y})$ are 2-dimensional representations of $\operatorname{Gal}(\overline{\mathbb{Q}} / \mathbb{Q})$. By a conjecture of Fontaine and Mazur [8] these representations should be modular, which, as we shall show, is indeed the case. To make a precise statement let $\eta(q)=q^{1 / 12} \prod_{n=1}^{\infty}\left(1-q^{n}\right)$ be the Dedekind $\eta$-function and define

$$
f(q):=\left[\eta(q) \eta\left(q^{2}\right) \eta\left(q^{3}\right) \eta\left(q^{6}\right)\right]^{2} .
$$

This is a (normalized) newform of weight 4 with respect to the group $\Gamma_{0}(6)$. In fact, one has $S_{4}\left(\Gamma_{0}(6)\right)=\mathbb{C} \cdot f$, where $S_{k}(\Gamma)$ denotes the vector space of cusp forms of weight $k$ with respect to a congruence group $\Gamma$. 
Theorem 3.2. There is an equality

$$
L\left(H_{\mathrm{et}}^{3}(Y), s\right) \stackrel{\circ}{=}\left(H_{\mathrm{et}}^{3}(\tilde{Y}), s\right) \stackrel{\circ}{=}(f, s)
$$

where $L_{1} \stackrel{\circ}{=} L_{2}$ means that the Euler factors of $L_{1}$ and $L_{2}$ coincide with the possible exception of the bad primes which are 2 and 3 in this case.

Remark 0.1. The rigidity of $Y$ and the modularity of $L\left(H_{\mathrm{et}}^{3}(\tilde{Y}), s\right)$ were already noted in [20, p. 864]. The $L$-function of $Y$ was also known to R. Livné (unpublished). See also Yui's survey paper $[24,5.4]$ where in addition the question was raised whether there is a connection with Verrill's Calabi-Yau threefold.

Using the $2: 1$ cover $\tilde{Y} \rightarrow Y$ the proof of this theorem can be reduced to checking that $L\left(H_{\text {ét }}^{3}(Y), s\right) \stackrel{\circ}{=}(f, s)$. By a result due to Serre it suffices to check equality of the Fourier coefficients $a_{p}$ for the primes $p=5,7,11,13,17,19,23$ and 73. (These primes are good primes which represent the elements of $(\mathbb{Z} / 24 \mathbb{Z})^{*}$.)

Given any (normalized) newform $g$ of weight 4 with respect to $\Gamma_{0}(N)$ with Fourier coeffcients in $\mathbb{Z}$, there are 2 -dimensional $\ell$-adic $\operatorname{Gal}(\overline{\mathbb{Q}} / \mathbb{Q})$ representations $\rho_{g, \ell}$ on $\mathbb{Q}_{\ell}^{2}$ with the property that $L\left(\rho_{g, \ell}\right)=L(g, s)$. The existence of these Galois representations was established by Deligne, and they occur naturally in the étale cohomology of the fibre square $W_{N}:=S_{1}(N) \times_{X_{1}(N)} S_{1}(N)$ of the universal elliptic curve $S_{1}(N)$ over the modular curve $X_{1}(N)$. In our case we have the Shimura isomorphism $S_{4}\left(\Gamma_{0}(6)\right) \cong$ $S_{4}\left(\Gamma_{1}(6)\right) \cong H^{3,0}(\hat{W})$ where $\hat{W}$ is a resolution of

$$
W:=W_{6}=S_{1}(6) \times_{X_{1}(6)} S_{1}(6)
$$

(cf. [16]). The Galois representations $\rho_{f, \ell}$ are subrepresentations of $H_{\text {ét }}^{3}\left(\hat{W}, \mathbb{Q}_{\ell}\right)$.

The Tate conjecture implies that if isomorphic Galois representations $\rho_{1}, \rho_{2}$ occur in the étale cohomology of varieties $X_{1}, X_{2}$ defined over $\mathbb{Q}$, then there is a correspondence, defined over $\mathbb{Q}$, between $X_{1}$ and $X_{2}$ which induces an isomorphism between $\rho_{1}$ and $\rho_{2}$.

We say that a smooth projective variety $X$, defined over $\mathbb{Q}$, is a relative of the Barth-Nieto quintic $N$ if the Galois representation $\rho_{f, \ell}$ occurs in $H_{\text {ét }}^{3}\left(X_{\overline{\mathbb{Q}}}\right)$. Tate's conjecture implies that relatives should be in correspondence. Since this conjecture is still very much open, we tried (and succeeded!) in establishing some correspondences.

The relative $\tilde{Y}$ is birationally equivalent to $\tilde{N}$, the double cover of $N$, and thus there are correspondences between these relatives. Using the explicit equations for the varieties involved we also find a correspondence between $\tilde{Y}$ and $\hat{W}$ :

Theorem 4.1. There exists a birational equivalence between $S_{1}(6) \times_{X_{1}(6)} S_{1}(6)$ and $Y$ which is defined over $\mathbb{Q}$.

In her investigations of rigid Calabi-Yau threefolds $\mathrm{H}$. Verrill met another relative of the Barth-Nieto quintic. It is a threefold denoted by $\mathscr{Z}_{A_{3}}$ and it is constructed using the root system $A_{3}$. We establish a correspondence with this relative: 
Theorem (cf. Theorem 4.3). There is a birational equivalence between $Y$ and $\mathscr{Z}_{A_{3}}$ which is defined over $\mathbb{Q}$.

Combining this theorem with Theorem 4.1 gives a birational equivalence between $\mathscr{Z}_{A_{3}}$ and $S_{1}(6) \times_{X_{1}(6)} S_{1}(6)$. Such an equivalence was first found by M. Saito and $\mathrm{N}$. Yui (see [16]), but their equivalence is different from ours.

Acknowledgement. We thank R. Livné and Ch. Schoen for helpful comments.

\section{The two protagonists}

In this section we recall the construction of our two protagonists $Y$ and $Z$ from [1].

The construction of the Calabi Yau varieties $Y$ and $Z$ from the Nieto quintic $N$ is summarized by the following commutative diagram:

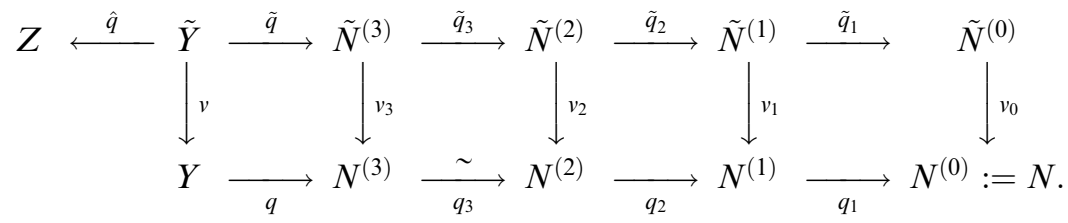

The three squares on the right are derived from the analogous diagram

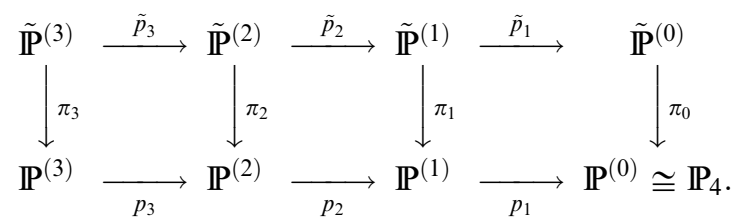

The varieties appearing in the first (second) diagram have dimension three (four). The horizontal maps are birational, the vertical maps are generically $2: 1$.

We now describe the diagrams in detail. We work in

$$
\mathbb{P}^{(0)}:=\left\{x \in \mathbb{P}_{5}: x_{0}+\cdots+x_{5}=0\right\} \cong \mathbb{P}_{4}
$$

The Nieto quintic is the irreducible hypersurface

$$
N^{(0)}:=N:=\left\{x \in \mathbb{P}^{(0)}: \sigma_{5}(x)=0\right\}
$$

where

$$
\sigma_{5}(x):=\sum_{j=0}^{5} x_{0} \ldots \hat{x}_{i} \ldots x_{5}=x_{0} \ldots x_{5} \sum_{j=0}^{5} \frac{1}{x_{j}}
$$


Its singular locus consists of the 20 lines

$$
L_{k \ell m}:=\left\{x_{k}=x_{\ell}=x_{m}=0\right\} \quad(0 \leqslant k<\ell<m \leqslant 5)
$$

in $\mathbb{P}^{(0)}$ and the 10 "Segre" points

$$
(1: 1: 1:-1:-1:-1)+\text { permutations. }
$$

These Segre points are ordinary double points of $N$. The lines $L_{k \ell m}$ intersect in the 15 points

$$
P_{k \ell m n}=\left\{x_{k}=x_{\ell}=x_{m}=x_{n}=0\right\} \quad(0 \leqslant k<\ell<m<n \leqslant 5) .
$$

We also need the 15 planes

$$
F_{k \ell}:=\left\{x_{k}=x_{\ell}=0\right\} \quad(0 \leqslant k<\ell \leqslant 5)
$$

and the 6 hyperplanes

$$
S_{k}=\left\{x_{k}=0\right\} \quad(0 \leqslant k \leqslant 5) .
$$

Note that

$$
S_{k} \cap N=\bigcup_{\ell=0}^{5} F_{k \ell} .
$$

The map $p_{1}$ is the blow up in the 15 points $P_{k \ell m n}$. The map $p_{2}$ is the blow up along the 20 lines $L_{k \ell m}^{(1)}$, where $L_{k \ell m}^{(1)} \subset \mathbb{P}^{(1)}$ denotes the strict transform of $L_{k \ell m}$ under $p_{1}$. More generally, we denote by $L_{k \ell m}^{(i)}, F_{k \ell}^{(i)}, \ldots$ the strict transform of $L_{k \ell m}, F_{k \ell}, \ldots$ in $\mathbb{P}^{(i)}$. Note that the lines $L_{k \ell m}^{(1)}$ are disjoint. Finally, the map $p_{3}$ is the blow up along the 15 disjoint "planes" $F_{k \ell}^{(2)}$. Note that $F_{k \ell}^{(2)} \cong \hat{\mathbb{P}}_{2}(6)$, a plane blown up in 6 points.

We define $N^{(i)}$ to be the strict transform of the Nieto quintic in $\mathbb{P}^{(i)}$ and we set $q_{i}:=\left.p_{i}\right|_{N^{(i)}}$. Note that $q_{3}$ is an isomorphism. It was shown in [1] that the only singularities of $N^{(3)} \cong N^{(2)}$ are the 10 Segre points. The Calabi-Yau model $Y$ of $N$ is a small resolution of $N$, i.e. the map $q: Y \rightarrow N^{(3)}$ replaces 10 points by 10 lines.

We now describe the $2: 1$-coverings. The map $\pi_{i}: \tilde{\mathbb{P}}^{(i)} \rightarrow \mathbb{P}^{(i)}$ is the double cover with branch locus $D_{i}$, where

$$
D_{0}:=\sum_{k=0}^{5} S_{k} \subseteq \mathbb{P}^{(0)}
$$

and $D_{i}$ is the odd part of the pull back of $D_{0}$ in $\mathbb{P}^{(i)}$. Explicitely, denoting the exceptional divisor of $p_{i}$ in $\mathbb{P}^{(i)}$ by $E_{i}$ and its strict transform in $\mathbb{P}^{(j)}(j>i)$ by $E_{i}^{(j)}$, we have 
- $p_{1}^{*}\left(D_{0}\right)=\sum S_{k}^{(1)}+4 E_{1}$, whence $D_{1}=\sum S_{k}^{(1)}$;

- $p_{2}^{*}\left(D_{1}\right)=\sum S_{k}^{(2)}+3 E_{2}$, whence $D_{2}=\sum S_{k}^{(2)}+E_{2}$;

- $p_{3}^{*}\left(D_{2}\right)=\sum S_{k}^{(3)}+E_{2}^{(3)}+2 E_{3}$, whence $D_{3}=\sum S_{k}^{(3)}+E_{2}^{(3)}$.

Remark 1.1. Note that $D_{3}$ and $\tilde{\mathbb{P}}^{(3)}$ are singular, since $S_{k}^{(3)} \cap E_{2}^{(3)} \neq \varnothing$, even though Barth and Nieto claim they are smooth [1, p. 216]. This problem disappears after restricting to $N^{(3)}$, since $S_{k}^{(3)} \cap N^{(3)}=\varnothing$. On the other hand, $S_{k}^{(2)} \cap N^{(2)} \neq \varnothing$, so the double cover $v_{3}: \tilde{N}^{(3)} \rightarrow N^{(3)} \cong N^{(2)}$ is different from $v_{2}: \tilde{N}^{(2)} \rightarrow N^{(2)}$.

Finally, we let $\tilde{N}^{(i)}:=N^{(i)} \times_{\mathbb{P}^{(i)}} \tilde{\mathbb{P}}^{(i)}$. The singular locus of $\tilde{N}^{(3)}$ consists of 20 double points and $\tilde{q}: \tilde{Y} \rightarrow \tilde{N}^{(3)}$ is a small resolution. The branch divisor $D_{3} \cap N^{(3)}=$ $E_{2}^{(3)} \cap N^{(3)}$ of $v_{3}: \tilde{N}^{(3)} \rightarrow N^{(3)}$ consists of 20 disjoint quadrics. The map $\hat{q}: \tilde{Y} \rightarrow Z$ contracts these quadrics to 20 lines.

\section{The topology of $Y$ and $Z$}

In this section we prove the following

Theorem 2.1. Both $Y$ and $Z$ have Hodge numbers $h^{0,0}=h^{3,0}=h^{0,3}=h^{3,3}=1$ and $h^{1,0}=h^{0,1}=h^{2,0}=h^{0,2}=h^{2,1}=h^{1,2}=h^{3,1}=h^{1,3}=h^{3,2}=h^{2,3}=0$. In particular, both manifolds are rigid. Furthermore, $h^{1,1}(Y)=h^{2,2}(Y)=50$ whereas $h^{1,1}(Z)=$ $h^{2,2}(Z)=40$.

Remark 2.2. This holds for any smooth Calabi-Yau model of $Y$ or $Z$, since birational smooth Calabi-Yau varieties have the same Betti numbers [2]. (Note that the Hodge numbers of a smooth Calabi-Yau variety of dimension 3 are determined by its Betti numbers.)

Recall that $Y$ and $Z$ are smooth Calabi-Yau varieties of dimension 3. This determines the boundary of the Hodge diamond: $h^{0,0}=h^{3,0}=h^{0,3}=h^{3,3}=1$ and $h^{1,0}=$ $h^{0,1}=h^{2,0}=h^{0,2}=h^{3,1}=h^{1,3}=h^{3,2}=h^{2,3}=0$. The remaining Hodge numbers are determined by

$$
a:=h^{2,1}(Y) \quad \text { and } \quad b:=h^{2,1}(Z)
$$

and the respective Euler characteristics $e(Y)=100$ and $e(Z)=80$. Indeed, we have $h^{2,1}(Y)=h^{1,2}(Y)=a, \quad h^{2,1}(Z)=h^{1,2}(Z)=b, \quad h^{1,1}(Y)=h^{2,2}(Y)=a+50$ and $h^{1,1}(Z)=h^{2,2}(Z)=b+40$.

It remains to show that $a=b=0$. This is done in the remainder of this long section. (A short roadmap is given at the end of the next subsection.) As mentioned in the introduction, we use the reductions of our varieties over suitable finite fields. We now explain this "reduction method" in detail. 
2.1 The reduction method. We first deal with $Y$. Note that $Y$ is defined over $\mathbb{Z}$. We write $\mathbb{F}_{p}:=\mathbb{Z} / p \mathbb{Z}$; more generally, we write $\mathbb{F}_{q}$ for the finite field with $q$ elements.

Lemma 2.3. If $p$ is prime and $p \geqslant 5$, then the reduction of $Y$ modulo $p$ is smooth over $\mathbb{F}_{p}$.

Proof. This follows from an easy calculation (cf. [1, (3.1), (9.1) and (9.3)]).

From now on, $p$ denotes a prime number $\geqslant 5$. Let $Y\left(\mathbb{F}_{p}\right)$ be the set of points of $Y$ which are rational over $\mathbb{F}_{p}$. By Grothendieck's version of the Lefschetz fixed point formula, we have

$$
\# Y\left(\mathbb{F}_{p}\right)=\sum_{j=0}^{6}(-1)^{j} \operatorname{tr}\left(\operatorname{Frob}_{p}^{*} ; H_{\text {èt }}^{j}(Y)\right) \text {, }
$$

where Frob $_{p}: Y \rightarrow Y$ is the Frobenius morphism [12, p. 454]. Using the abbreviation

$$
t_{j}:=\operatorname{tr}\left(\operatorname{Frob}_{p}^{*} ; H_{\text {ét }}^{j}(Y)\right)
$$

we have $t_{0}=1, t_{6}=p^{3}$ and $t_{1}=t_{5}=0$, whence

$$
\# Y\left(\mathbb{F}_{p}\right)=1+t_{2}-t_{3}+t_{4}+p^{3}
$$

The Weil conjectures assert that the eigenvalues of $\mathrm{Frob}_{p}$ on $H_{\text {ét }}^{j}(Y)$ are algebraic integers, which do not depend on $\ell$ and which have (archimedian) absolute value $p^{j / 2}$. This implies that the $t_{j}$ are ordinary integers, independent of $\ell$, with absolute value $\left|t_{j}\right| \leqslant b_{j}(Y) \cdot p^{j / 2}$. We use this only for $j=3$ :

$$
\left|t_{3}\right| \leqslant(2 a+2) p^{3 / 2}
$$

For $j=2$ we use the following stronger result, the proof of which we postpone to the end of this section.

Proposition 2.4. If $p \geqslant 5$ then all eigenvalues of $\mathrm{Frob}_{p}$ on $H_{\mathrm{e} t}^{2}(Y)$ are equal to $p$.

Hence $t_{2}=b_{2}(Y) p=(a+50) p$ and $t_{4}=(a+50) p^{2}$, by Poincaré duality. Putting everything together, we get

$$
\left|1+(a+50)\left(p+p^{2}\right)+p^{3}-\# Y\left(\mathbb{F}_{p}\right)\right| \leqslant(2 a+2) p^{3 / 2} .
$$

For $p=13$ we will find $\# Y\left(\mathbb{F}_{p}\right)=11260$, whence $182 a+38 \leqslant 94(a+1)$ implying $a=0$.

We use the same method to show that $b=0$. In fact, we work with $\tilde{Y}$ rather than $Z$. The reason is that it is not clear to us if the map $\hat{q}: \tilde{Y} \rightarrow Z$ is defined over $\mathbb{Z}$, 
since it was obtained in [1] using Mori theory. On the other hand, $\tilde{Y}$ is clearly defined over $\mathbb{Z}$. Furthermore, $h^{i, j}(\tilde{Y})=h^{i, j}(Z)$ for $(i, j) \neq(1,1)$ or $(2,2)$, whereas $h^{1,1}(\tilde{Y})=$ $h^{2,2}(\tilde{Y})=b+60$.

Lemma 2.5. If $p$ is prime and $p \geqslant 5$, then the reduction of $\tilde{Y}$ modulo $p$ is smooth over $\mathbb{F}_{p}$.

Proof. Just note that the proof of $[1,(10.2)]$ still works.

We also need the following proposition, which we will also prove at the end of this section.

Proposition 2.6. If $p \equiv 1 \bmod 4$ then all eigenvalues of $\mathrm{Frob}_{p}$ on $H_{\mathrm{ett}}^{2}(\tilde{Y})$ are equal to $p$.

So for $p \equiv 1 \bmod 4$ (which implies $p \geqslant 5$ ) we have

$$
\left|1+(b+60)\left(p+p^{2}\right)+p^{3}-\# \tilde{Y}\left(\mathbb{F}_{p}\right)\right| \leqslant(2 b+2) p^{3 / 2}
$$

For $p=13$ we will see that $\# \tilde{Y}\left(\mathbb{F}_{p}\right)=13080$, whence $182 b+38 \leqslant 94(b+1)$ implying $b=0$.

Summarizing, we have to determine the number of $\mathbb{F}_{13}$-rational points on $Y$ and $\tilde{Y}$, and we have to prove Propositions 2.4 and 2.6. This is done in $\S 2.3$ and $\$ 2.4$, respectively. The next subsection is a preparation for $\S 2.3$.

2.2 Counting points on Cayley cubics. In order to count the points of $Y\left(\mathbb{F}_{p}\right)$ we will need to understand the structure of the exceptional divisor in $Y$ lying over a point $P_{k \ell m n}$. We now collect the necessary information. Since $Y \rightarrow N^{(3)} \cong N^{(2)}$ is an isomorphism outside the Segre points, we can consider $N^{(2)}$ instead of $Y$ : the fibres over $P_{k \ell m n}$ are the same. One easily checks (see [1, (9.1)] and the proof of 2.7 below) that the fibre of $N^{(1)} \rightarrow N$ lying over $P_{0123}=(0: 0: 0: 0:-1: 1)$ is the Cayley cubic

$$
C^{(1)}: \frac{1}{y_{0}}+\frac{1}{y_{1}}+\frac{1}{y_{2}}+\frac{1}{y_{3}}=0
$$

in $\mathbb{P}_{3}$. This $\mathbb{P}_{3}$ is of course the component of $E_{1} \subset \mathbb{P}^{(1)}$ lying over $P_{0123}$ and $C^{(1)}=$ $\mathbb{P}_{3} \cap N^{(1)}$.

Analogously, in order to determine $\# \tilde{Y}\left(\mathbb{F}_{p}\right)$ we need information about the fibre of $\tilde{Y} \rightarrow N$ lying over $P_{k \ell m n}$. Since $\tilde{Y} \rightarrow \tilde{N}^{(3)}$ is an isomorphism outside the Segre points, we may replace $\tilde{Y}$ by $\tilde{N}^{(3)}$. Now recall that the double cover $\tilde{N}^{(3)} \rightarrow N^{(3)} \cong$ $N^{(2)}$ is branched along the exceptional divisor $E_{2} \cap N^{(2)}$ of the blow up $N^{(2)} \rightarrow N^{(1)}$ along the lines $L_{k \ell m n}^{(1)}$. Hence $\tilde{C}^{(2)}$ is the double cover of $C^{(2)}$ branched along $E_{2} \cap$ $C^{(2)}$, which consists of the four exceptional lines of $C^{(2)} \rightarrow C^{(1)}$. 
Lemma 2.7. Let $p \equiv 1 \bmod 4$. Then $\# \tilde{C}^{(3)}\left(\mathbb{F}_{p}\right)=p^{2}+8 p+1$.

Proof. First we determine the blow up of $N$ in the point

$$
P_{0123}=(0: 0: 0: 0:-1: 1)
$$

We blow up the $\mathbb{A}_{5} \subset \mathbb{P}_{5}$ where $x_{5}=1$ in $P=(0,0,0,0,-1)$, this is the subvariety of $\mathbb{A}_{5} \times \mathbb{P}_{4}$ defined by

$$
x_{i} y_{j}-x_{j} y_{i}=0, \quad\left(x_{4}+1\right) y_{j}-x_{j} y_{4}=0, \quad 0 \leqslant i<j \leqslant 3 .
$$

The inverse image of $N \cap \mathbb{A}_{5}$ in the open set $\mathbb{A}_{5} \times \mathbb{A}_{4}$ defined by $y_{4}=1$ is given by the equations $x_{j}=\left(x_{4}+1\right) y_{j}$, so we are left with the variables $x_{4}, y_{0}, \ldots, y_{3}$, the equation

$$
\left(x_{4}+1\right)\left(y_{0}+y_{1}+y_{2}+y_{3}+1\right)=0
$$

(obtained from $\left.\sum x_{i}=0\right)$ and the equation

$$
\left(x_{4}+1\right)^{3}\left(y_{0} y_{1} y_{2} y_{3}\left(x_{4}+1\right)^{2}+x_{4}\left(y_{0} y_{1} y_{2}+y_{0} y_{1} y_{3}+y_{0} y_{2} y_{3}+y_{1} y_{2} y_{3}\right)\right)=0
$$

(obtained from $\sigma_{5}=x_{0} x_{1} x_{2} x_{3} x_{4}+\cdots+x_{1} x_{2} x_{3} x_{4} x_{5}=0$ ). The fibre over $P$ is defined by $x_{4}=-1$ and we see that the fibre over $P_{0123}$ in the blow up $N^{(1)} \rightarrow N$ is the Cayley cubic $C^{(1)}$ in a $\mathbb{P}_{3}$ (note $y_{4}=-\sum y_{i}$ ) with coordinates $y_{0}, \ldots, y_{3}$.

The double cover $\tilde{N}$ of $N$ ramifies over the zero locus of $x_{0} x_{1} x_{2} x_{3} x_{4} x_{5}$. One can thus construct it naturally in a weighted projective space. Equivalently, consider the image of $N$ in $\mathbb{P}_{k}$ under the third Veronese map, so the coordinates on $\mathbb{P}_{k}$ are the $u_{i j k}$ with $u_{i j k}=x_{i} x_{j} x_{k}$. Then $\tilde{N}$ is the subvariety of $\mathbb{P}_{k+1}$, with coordinates $u_{i j k}$ and $v$, defined by the equations of the image of $N$ in $\mathbb{P}_{k}$ and the equation $v^{2}=u_{012} u_{345}$. The inverse image of $N \cap \mathbb{A}_{5}$ in $\tilde{N}$ is therefore isomorphic to the subvariety of $\mathbb{A}_{6}$, with coordinates $x_{0}, \ldots, x_{4}$ and $v$, defined by the equations of $N \cap \mathbb{A}_{5}$ and the equation $v^{2}=x_{0} x_{1} x_{2} x_{3} x_{4}$.

To obtain $\tilde{N}^{(1)}$, locally near $P_{0123}$, we consider the inverse image of $N \cap \mathbb{A}_{5}$ in the open set of the blow up where $y_{4}=1$ :

$$
v^{2}=\left(x_{4}+1\right)^{4} x_{4} y_{0} y_{1} y_{2} y_{3}
$$

As explained in Section 1, we need to ramify only over the odd part of this divisor, so locally $\tilde{N}^{(1)}$ is defined by

$$
w^{2}=x_{4} y_{0} y_{1} y_{2} y_{3}
$$

and the equations defining the strict transform of $N \cap \mathbb{A}_{5}$. Restricted to the special fibre $x_{4}=-1$ this gives the equation $w^{2}=-y_{0} y_{1} y_{2} y_{3}$. In case -1 is a square in the 
field under consideration (for example $\mathbb{F}_{p}$ with $p \equiv 1 \bmod 4$ ), we can change the variable to obtain the equation $w_{1}^{2}=y_{0} y_{1} y_{2} y_{3}$.

The equation $y_{i}=0$ defines the divisor $S_{i}^{(1)}$. The intersection $S_{i}^{(1)} \cap C^{(1)}$ consists of three lines in $C^{(1)}$. In this way we get the 6 lines $S_{i}^{(1)} \cap S_{j}^{(1)} \cap C^{(1)}$ in the Cayley cubic which connect the nodes. In particular, the cover $\tilde{C}^{(1)} \rightarrow C^{(1)}$ ramifies over these 6 lines and these lines are singular on $\tilde{C}^{(1)}$.

The map $C^{(2)} \rightarrow C^{(1)}$ is the blow up of the Cayley cubic in the 4 nodes. We denote the exceptional fibres, $(-2)$-curves, by $R_{i}^{(2)}$. The double cover $\tilde{C}^{(2)} \rightarrow C^{(2)}$ ramifies along the 6 lines and the $4 R_{i}^{(2)}$ 's. The map $N^{(3)} \rightarrow N^{(2)}$ is an isomorphism (since the $F_{k l}^{(2)}$ are smooth surfaces in the smooth part of the threefold $\left.N^{(2)}\right)$. But since $F_{k l}^{(1)}=$ $S_{k}^{(1)} \cap S_{l}^{(1)}$ intersects the Cayley cubic in a line, the map $\tilde{C}^{(3)} \rightarrow \tilde{C}^{(2)}$ is the normalization of $\tilde{C}^{(2)}$. Therefore the double cover

$$
\tilde{C}^{(3)} \rightarrow C^{(3)}=C^{(2)}
$$

ramifies only over the four nodal curves $R_{i}$. It is easy to check that $h^{1,0}\left(\tilde{C}^{(3)}\right)=$ $h^{2,0}\left(\tilde{C}^{(3)}\right)=0$ and $h^{1,1}\left(\tilde{C}^{(3)}\right)=8$.

We recall the explicit parametrizations for the Cayley cubic and its double cover, ramified only in the nodes. The cubic $C^{(1)}$ is obtained by blowing up a $\mathbb{P}_{2}$ in 6 points which are the intersection points of 4 general lines, next one blows down the strict transforms of the 4 lines. For the plane we will take

$$
\mathbb{P}_{2}:=\left\{x \in \mathbb{P}_{3}: x_{0}+x_{1}+x_{2}+x_{3}=0\right\},
$$

the 4 lines are defined by $x_{i}=0$ so the 6 points $x_{i}=x_{j}=0(0 \leqslant i<j \leqslant 3)$. The linear system of cubics through these 6 points is generated by $x_{0} x_{1} x_{2}, x_{0} x_{1} x_{3}, x_{0} x_{2} x_{3}$, and $x_{1} x_{2} x_{3}$. Denoting temporarily the coordinates of the target space $\mathbb{P}_{3}$ by $\left(y_{0}\right.$ : $\left.y_{1}: y_{2}: y_{3}\right)$, we see that the associated rational map $\mathbb{P}_{2} \rightarrow \mathbb{P}_{3},\left(x_{0}: x_{1}: x_{2}: x_{3}\right) \mapsto$ $x_{0} x_{1} x_{2} x_{3}\left(\frac{1}{x_{0}}: \frac{1}{x_{1}}: \frac{1}{x_{2}}: \frac{1}{x_{3}}\right)=:\left(y_{0}: y_{1}: y_{2}: y_{3}\right)$, maps $\mathbb{P}_{2}$ to the Cayley cubic $\sum \frac{1}{y_{i}}=$ 0 . It blows up the six points, the exceptional divisors being the edges of the tetrahedron spanned by the four singular points of $C^{(1)}$, and it contracts the four lines $x_{i}=0$ to these singular points. In other words, the map $\mathbb{P}_{2} \rightarrow C^{(1)}$ induces an isomorphism

$$
\hat{\mathbb{P}}_{2}(6) \stackrel{\sim}{\rightarrow} C^{(2)},
$$

where $\hat{\mathbb{P}}_{2}(6)$ is the blow up of $\mathbb{P}_{2}$ in the 6 points. This also works over the finite field $\mathbb{F}_{p}$, hence $\# C^{(2)}\left(\mathbb{F}_{p}\right)=1+7 p+p^{2}$.

An alternative description of $\tilde{C}^{(3)}$ would be the double cover of $\mathbb{P}_{2}$ branched along the four lines $x_{i}=0$ with the six quadratic singular points lying over the intersection points resolved by a $(-2)$-curve.

Since each of the 6 points lies on exactly two lines, the inverse image in $\tilde{C}^{(3)}$ of the exceptional divisor is an irreducible rational curve which maps $2: 1$ onto the exceptional divisor in $C^{(3)}$. Note that the line $x_{3}+x_{4}=0$ is parametrized by 
$(s:-s: t:-t)$ and splits in this double cover since it meets the ramification locus only in the points $(1:-1: 0: 0)$ and $(0: 0: 1:-1)$ and each point has multiplicity 2 .

Consider the morphism $f: C^{(3)} \rightarrow \mathbb{P}_{1}$ defined by the pencil of lines through the point $(1:-1: 0: 0)$ (so $f$ is obtained from the rational map $\mathbb{P}_{3} \rightarrow \mathbb{P}_{1}$, $\left.\left(x_{0}: x_{1}: x_{2}: x_{3}\right) \mapsto\left(x_{2}: x_{3}\right)\right)$. If the line in the pencil $f$ meets the ramification locus in two other distinct points, besides $(1:-1: 0: 0)$, the corresponding fibre of the composite map $\tilde{f}: \tilde{C}^{(3)} \rightarrow C^{(3)} \rightarrow \mathbb{P}_{1}$ is smooth. There are only three exceptions: the lines $x_{2}=0, x_{3}=0$ and $x_{3}+x_{4}=0$.

The fibre of $\tilde{f}$ corresponding to the line $x_{3}=0$ consists of the strict tranform of this line as well the two $(-2)$-curves corresponding to the points $(1: 0:-1: 0)$ and $(0: 1:-1: 0)$. Thus $\tilde{f}^{-1}(1: 0)$ is a tree of three $\mathbb{P}_{1}$ 's, and the same holds for $\tilde{f}^{-1}(0: 1)$. We already observed that the line $x_{3}+x_{4}=0$ splits in the covering. Besides these two components, $f^{-1}(1:-1)$ also contains the $(-2)$-curve over the point $(0: 0: 1:-1)$ and this fibre is thus also a tree of three $\mathbb{P}_{1}$ 's.

If the two components over the line $x_{3}+x_{4}=0$ are rational over $\mathbb{F}_{p}$, then the number of points of $\tilde{C}^{(3)}$ over $\mathbb{P}_{1}$ minus the three exceptional points is $(p-2)(p+1)$, and each exceptional point contributes a tree of three $\mathbb{P}_{1}$ 's hence $3(p+1)-2=$ $3 p+1$ points. Thus for such fields

$$
\# \tilde{C}^{(3)}\left(\mathbb{F}_{p}\right)=\left(p^{2}-p-2\right)+3(3 p+1)=p^{2}+8 p+1
$$

From this one easily obtains that $\operatorname{dim} H^{2}\left(\tilde{C}^{(3)}\right)=8$. (On $\tilde{C}^{(3)}$ we have 7 obviously independent divisor classes, the pull-back of a hyperplane section of $C^{(3)}$ and the 6 nodal curves. Each of the components over the line $x_{3}+x_{4}=0$ can serve as an "eighth divisor". To see this, one checks that the $8 \times 8$ intersection matrix has nonzero determinant.)

To see for which primes the two components over $x_{3}+x_{4}=0$ are rational over $\mathbb{F}_{p}$, we pull-back the defining equation for the double cover $w^{2}=-y_{0} y_{1} y_{2} y_{3}$ (and $y_{4}=$ $\left.-\left(y_{0}+\cdots+y_{3}\right)\right)$ to $\mathbb{P}_{2}$, and one finds that that over $(1:-1: t:-t)$ the double cover is given by $w^{2}=-t^{2}$, hence the two components are rational iff -1 is a square in the field iff $p \equiv 1 \bmod 4$.

Remark 2.8. For completeness sake we give the well-known identification of $\tilde{C}^{(3)}$ with a $\mathbb{P}^{2}$ blown up in 7 points. The standard Cremona transformation on $\mathbb{P}_{2},\left(t_{0}: t_{1}: t_{2}\right)$ $\mapsto\left(1 / t_{0}: 1 / t_{1}: 1 / t_{2}\right)$, is well defined on the blow up of $\mathbb{P}_{2}$ in the three 'basis' points and has 4 fixed points:

$$
(1: 1: 1), \quad(1: 1:-1), \quad(1:-1: 1), \quad(-1: 1: 1)
$$

Blowing up these 7 points gives a surface with $1+p+p^{2}+7 p=1+8 p+p^{2}$ points. The smooth quotient surface has $4(-2)$-curves (the images of the exceptional divisors over the fixed points) and blowing these down you get the Cayley cubic $C^{(1)}$. Therefore $\tilde{C}^{(3)}$ is isomorphic to the blowup of $\mathbb{P}_{2}$ in 7 points. An explict formula for 
the rational $2: 1$ map from $\mathbb{P}_{2}$ to $C^{(1)}$ is given by the following polynomials, which are invariant (modulo the common factor $\left(t_{0} t_{1} t_{2}\right)^{2}$ ) under the Cremona transformation

$$
x_{0}=2 t_{0} t_{1} t_{2}, \quad x_{1}=t_{0}\left(t_{1}^{2}+t_{2}^{2}\right), \quad x_{2}=t_{1}\left(t_{0}^{2}+t_{2}^{2}\right), \quad x_{3}=t_{2}\left(t_{0}^{2}+t_{1}^{2}\right)
$$

and they satisfy the cubic relation $2 x_{1} x_{2} x_{3}-x_{0}\left(x_{1}^{2}+x_{2}^{2}+x_{3}^{2}-x_{0}^{2}\right)$. The fixed points map to $(1: 1: 1: 1),(1: 1:-1:-1),(1:-1: 1:-1)$ and $(1:-1:-1: 1)$. Using the linear combinations $x_{0}+x_{1}+x_{2}+x_{3}, x_{0}-x_{1}+x_{2}-x_{3}, x_{0}+x_{1}-x_{2}-x_{3}$ and $x_{0}-x_{1}-x_{2}+x_{3}$, one obtains functions which satisfy the equation defining $C^{(1)}$.

Corollary 2.9. All eigenvalues of Frob $_{p}$ acting on $H_{\text {ét }}^{2}\left(\tilde{C}^{(2)}\right)$ are equal to $p$ if $p \equiv$ $1 \bmod 4$.

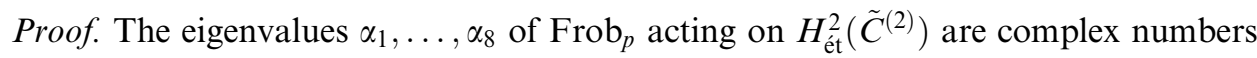
of absolute value $p$. Since $\alpha_{1}+\cdots+\alpha_{8}=8 p$ by the previous lemma, we find that $\alpha_{i}=p$ for $i=1, \ldots, 8$.

Remark 2.10. For $p \equiv 3 \bmod 4$ one finds $\# \tilde{C}^{(2)}\left(\mathbb{F}_{p}\right)=p^{2}+6 p+1$. Thus the eigenvalues of Frob $_{p}$ acting on $H_{\text {ét }}^{2}\left(\tilde{C}^{(2)}\right)$ are $p$ with multiplicity 7 and $-p$ with multiplicity 1.

Lemma 2.11. If $p \equiv 1 \bmod 4$ then the contribution over the unramified part of $F_{k \ell}$ to $\# \tilde{Y}\left(\mathbb{F}_{p}\right)$ is $p^{2}-2 p+3$.

Proof. We claim that the equation of the unramified part of the double cover of $F_{k \ell}$ is $y^{2}=-x_{0} x_{1} x_{2} x_{3}$ as in Lemma 2.7. In order to see this, we blow up $\mathbb{P}^{(0)}$ along $F_{k \ell}$. The resulting exceptional divisor is $\mathbb{P}_{1} \times F_{k \ell}$ and one easily checks that the strict transform of $N$ intersects this divisor in $\{(1:-1)\} \times F_{k \ell}$ with respect to the coordinates on the fibre $\mathbb{P}_{1}$ given by $x_{k}$ and $x_{\ell}$. On $\{(1:-1)\} \times F_{k \ell}$ we have $x_{k}=-x_{\ell}$, so restricting $x_{0} \ldots x_{5}$ and dividing by the square $x_{k}^{2}=x_{\ell}^{2}$ yields the desired $-x_{0} \ldots x_{5} / x_{k} x_{\ell}$.

Now we can apply Lemma 2.7. Indeed, the ramification locus, which consists of 6 intersecting lines, contains $10 p-2$ points over $\mathbb{F}_{p}$, the proper double cover $\tilde{F}_{k \ell} \cong$ $\tilde{C}^{(2)}$ of $F_{k \ell}$ contains $p^{2}+8 p+1$ points, leaving $\left(p^{2}+8 p+1\right)-(10 p-2)$ points for the unramified part.

Remark 2.12. The corresponding result for $p \equiv 3 \bmod 4, p \neq 3$, is $p^{2}-4 p+3$.

2.3 Counting points on $\boldsymbol{Y}$ and $\tilde{\boldsymbol{Y}}$. We will now show how to count the points of $Y$ and $\tilde{Y}$ over $\mathbb{F}_{p}$. Recall that $D_{0}=\left\{x_{0} \ldots x_{5}=0\right\}$. We set

$$
U:=N \backslash D_{0} .
$$

It is easy to count the points of $U\left(\mathbb{F}_{p}\right)$ on a computer. For $p=13$ one finds $\# U\left(\mathbb{F}_{13}\right)=2140$. Together with the following proposition this yields $\# Y\left(\mathbb{F}_{13}\right)=$ 11260. 
Proposition 2.13. For $p \geqslant 5$ we have $\# Y\left(\mathbb{F}_{p}\right)=\# U\left(\mathbb{F}_{p}\right)+50 p^{2}+50 p+20$.

Proof. Recall that $N \cap D_{0}$ is the union of the 15 planes $F_{k \ell}$. The map $Y \rightarrow N$ replaces the 10 Segre points by 10 lines, the 20 lines $L_{k \ell m}$ by quadrics and the 15 points $P_{k \ell m n}$ by smooth cubics $C^{(2)} \cong \hat{\mathbb{P}}_{2}(6)$. The Segre points lie in $U$, so here we get $10 p$ new points over $\mathbb{F}_{p}$. Over $R^{0}:=\bigcup F_{k \ell} \backslash \bigcup L_{k \ell m}$ nothing happens. Now $R^{0}$ consists of 15 copies of $\mathbb{P}_{2} \backslash(4$ lines $)$, hence

$$
\# R^{0}\left(\mathbb{F}_{p}\right)=15\left[\left(p^{2}+p+1\right)-4(p+1)+6\right]=15\left(p^{2}-3 p+3\right) .
$$

Similarly, $\mathscr{L}^{0}:=\bigcup L_{k \ell m} \backslash \bigcup P_{k \ell m n}$ consists of 20 copies of $\mathbb{P}_{1} \backslash(3$ points $)$. Now $Y \rightarrow N$ replaces $\mathscr{L}^{0}$ by $\mathscr{L}^{0} \times \mathbb{P}_{1}$ and its contribution to $\# Y\left(\mathbb{F}_{p}\right)$ is

$$
\#\left(\mathscr{L}^{0} \times \mathbb{P}_{1}\right)\left(\mathbb{F}_{p}\right)=20(p-2)(p+1)
$$

Finally, we have a copy of $\hat{\mathbb{P}}_{2}(6)$ over each of the 15 points $P_{k \ell m n}$, contributing

$$
15\left(p^{2}+7 p+1\right)
$$

Hence

$$
\begin{aligned}
\# Y\left(\mathbb{F}_{p}\right)= & \# U\left(\mathbb{F}_{p}\right)+10 p+15\left(p^{2}-3 p+3\right) \\
& +20(p-2)(p+1)+15\left(p^{2}+7 p+1\right) \\
= & \# U\left(\mathbb{F}_{p}\right)+50 p^{2}+50 p+20
\end{aligned}
$$

Now we count the points of $\tilde{Y}\left(\mathbb{F}_{p}\right)$. Let $\tilde{U} \subset \tilde{Y}$ be the inverse image of $U \subset Y$. One can again easily count the points in $\tilde{U}\left(\mathbb{F}_{p}\right)$ using

$$
\# \tilde{U}\left(\mathbb{F}_{p}\right)=2 \cdot \#\left\{x \in U\left(\mathbb{F}_{p}\right) \mid x_{0} \ldots x_{5} \text { is a square in } \mathbb{F}_{p}\right\}
$$

and a computer. Together with the following proposition this yields the desired $\# \tilde{Y}\left(\mathbb{F}_{13}\right)=2 \times 1720+9640=13080$.

Proposition 2.14. If $p \equiv 1 \bmod 4$ then $\# \tilde{Y}\left(\mathbb{F}_{p}\right)=\# \tilde{U}\left(\mathbb{F}_{p}\right)+50 p^{2}+90 p+20$.

Proof. Note that the 20 Segre points in $\tilde{N}^{(3)}$ lie in $\tilde{N}^{(3)}\left(\mathbb{F}_{p}\right)$, since $1^{3} \cdot(-1)^{3}$ is a square in $\mathbb{F}_{p}$. Hence $\# \tilde{Y}\left(\mathbb{F}_{p}\right)=\# \tilde{N}^{(3)}\left(\mathbb{F}_{p}\right)+20 p$ and we have to show that $\# \tilde{N}^{(3)}\left(\mathbb{F}_{p}\right)=\# \tilde{U}\left(\mathbb{F}_{p}\right)+50 p^{2}+70 p+20$. Now recall that the branch locus of $\tilde{N}^{(3)} \rightarrow N^{(3)}=N^{(2)}$ is $E_{2}^{(2)}$, the union of 20 disjoint quadrics. The intersection of this branch locus with the resolved Cayley cubic $C^{(2)}$ in $N^{(2)}$ lying over a point $P_{k \ell m n}$ consists of the 4 exceptional lines of $C^{(2)} \rightarrow C^{(1)}$. We refer to the complement as the open Cayley cubic. The contribution of the ramification locus $E_{2}^{(2)}$ to $\# \tilde{N}^{(3)}\left(\mathbb{F}_{p}\right)$ 
is $20(p+1)^{2}$. By Lemma 2.7 each open Cayley cubic contributes $\left(p^{2}+8 p+1\right)-$ $4(p+1)=p^{2}+4 p-3$. Lemma 2.11 implies that the contribution of $R^{0}=$ $\bigcup F_{k \ell} \backslash \bigcup L_{k \ell m}$ equals $15\left(p^{2}-2 p+3\right)$. So we find $\# \tilde{N}^{(3)}\left(\mathbb{F}_{p}\right)-\# \tilde{U}\left(\mathbb{F}_{p}\right)=$ $20(p+1)^{2}+15\left(p^{2}+4 p-3\right)+15\left(p^{2}-2 p+3\right)=50 p^{2}+70 p+20$.

Remark 2.15. For $p \equiv 3 \bmod 4, p \neq 3$, one finds $\tilde{Y}\left(\mathbb{F}_{p}\right)=\# \tilde{N}^{(3)}\left(\mathbb{F}_{p}\right)=\# \tilde{U}\left(\mathbb{F}_{p}\right)+$ $20(p+1)^{2}+15\left(p^{2}+2 p-3\right)+15\left(p^{2}-4 p+3\right)=\# \tilde{U}\left(\mathbb{F}_{p}\right)+50 p^{2}+10 p+20$.

2.4 Proofs of Propositions 2.4 and 2.6. In the remainder of this section we prove Propositions 2.4 and 2.6 about the action of Frobenius on $H_{\mathrm{et}}^{2}(Y)$ and $H_{\mathrm{et}}^{2}(\tilde{Y})$. We say that a variety over $\mathbb{Z}$ satisfies condition $(*)_{p}$ for a prime $p$ if all eigenvalues of Frob $_{p}$ acting on the second étale cohomology are equal to $p$. If $(*)_{p}$ holds for every good prime $p$, then we say that the variety satisfies condition $(*)$; if $(*)_{p}$ holds for every good prime $p \equiv 1 \bmod 4$, then we say that the variety satisfies condition $(* *)$. So we have to show that $Y$ and $\tilde{Y}$ satisfy $(*)$ and $(* *)$, respectively. (Note, however, that for the proofs of $a=b=0$ above, we only used the fact that $Y$ and $\tilde{Y}$ satisfy condition $(*)_{p}$ for $p=13$.)

We start with $Y$. Recall the sequence of maps $Y \stackrel{q}{\rightarrow} N^{(3)}=N^{(2)} \stackrel{q_{2}}{\rightarrow} N^{(1)} \stackrel{q_{1}}{\rightarrow} N^{(0)}=$ $N \subset \mathbb{P}_{4}$. Since $q^{*}: H_{\mathrm{et}}^{2}\left(N^{(3)}\right) \rightarrow H_{\mathrm{et}}^{2}(Y)$ is an isomorphism, it suffices to check that condition $(*)$ holds for $N^{(3)}=N^{(2)}$. So the following lemma finishes the proof of Proposition 2.4.

Lemma 2.16. Condition $(*)$ holds for $N=N^{(0)}, N^{(1)}$ and $N^{(2)}=N^{(3)}$.

Proof. The condition holds for $N^{(0)}=N$, since $H_{\text {êt }}^{2}(N)=H_{\text {êt }}^{2}\left(\mathbb{P}_{4}\right)$ by the Lefschetz hyperplane theorem [9, Corollary I.9.4]. In order to lift this result to $N^{(1)}$, we use the spectral sequence

$$
E_{2}^{j, 2-j}=H_{\mathrm{èt}}^{j}\left(N^{(0)}, R^{2-j} q_{1, *}\left(\mathbb{Q}_{\ell}\right)\right) \Rightarrow H_{\mathrm{ett}}^{2}\left(N^{(1)}\right)
$$

associated to $q_{1}: N^{(1)} \rightarrow N^{(0)}$. Since condition $(*)$ is stable under extensions of Galois modules, it suffices to check the analogous condition on the graded parts $E_{2}^{j, 2-j}$ of $H_{\text {ét }}^{2}\left(N^{(1)}\right)$. Now we already know that Frobenius acts correctly on $E_{2}^{2,0}=$ $H_{\mathrm{ett}}^{2}\left(N^{(0)}\right)$. For $j=1$ we find $E_{2}^{1,1}=H_{\mathrm{et}}^{1}\left(\mathscr{P}, R^{1} q_{1, *}\left(\mathbb{Q}_{\ell}\right)\right)=0$, since the support $\mathscr{P}:=$ $\left\{P_{k \ell m n}\right\}$ of $R^{1} q_{1, *}\left(\mathbb{Q}_{\ell}\right)$ has dimension 0. Finally, we have to consider $E_{2}^{0,2}=$ $H_{\text {êt }}^{0}\left(\mathscr{P}, R^{2} q_{1, *}\left(\mathbb{Q}_{\ell}\right)\right)=H_{\text {et }}^{2}\left(C^{(1)}\right)^{\oplus 15}$. So it suffices to note that $C^{(1)}$ satisfies condition $(*)$. Indeed, $H^{2}\left(C^{(1)}\right)$ is a Galois submodule of $H^{2}\left(C^{(2)}\right)$ and the latter space is generated by a line and 6 exceptional divisors, all of which are obviously defined over $\mathbb{F}_{p}$.

The step from $N^{(1)}$ to $N^{(2)}$ is similar. Recall that $N^{(2)} \rightarrow N^{(1)}$ contracts 20 quadrics to lines. Hence we have a spectral sequence

$$
E_{2}^{j, 2-j}=H_{\mathrm{èt}}^{j}\left(N^{(1)}, R^{2-j} q_{2, *}\left(\mathbb{Q}_{\ell}\right)\right) \Rightarrow H_{\mathrm{ett}}^{2}\left(N^{(2)}\right)
$$

with $E_{2}^{2,0}=H_{\mathrm{et}}^{2}\left(N^{(1)}\right), E_{2}^{1,1}=0$ and $E_{2}^{0,2}=\oplus H_{\mathrm{et}}^{2}\left(L_{k \ell m}^{(1)}\right)=\mathbb{Q}_{\ell}(-1)^{\oplus 20}$. This proves the assertion for $N^{(2)}$. 
Finally, we prove Proposition 2.6, i.e. we check condition $(* *)$ for $\tilde{Y}$. Since the Leray spectral sequence of $\tilde{q}: \tilde{Y} \rightarrow \tilde{N}^{(3)}$ induces an exact sequence

$$
0 \rightarrow H_{\text {èt }}^{2}\left(\tilde{N}^{(3)}\right) \rightarrow H_{\text {èt }}^{2}(\tilde{Y}) \rightarrow H^{2}\left(\mathbb{P}_{1}\right)^{\oplus 20},
$$

where the $\mathbb{P}_{1}$ 's are the fibres over the Segre points, is suffices to show that $\tilde{N}^{(3)}$ satisfies $(* *)$. (Note that the $\mathbb{P}_{1}$ 's are defined over $\mathbb{F}_{p}$.) We use a Lefschetz argument relating $H_{\text {èt }}^{2}\left(\tilde{N}^{(3)}\right)$ to $H_{\text {èt }}^{2}(H)$ for a suitable divisor $H \subset \tilde{N}^{(3)}$. The complement $\tilde{N}^{(3)} \backslash H$ will contain the 20 Segre points, so we have to modify the hyperplane theorem to deal with them. This is done in the following lemma, which we will apply to $X=\tilde{N}^{(3)}$.

Lemma 2.17. Let $X$ be a projective threefold. Let $H \subset X$ be a divisor such that $X \backslash H$ is affine and $\Sigma:=\operatorname{Sing}(X \backslash H)$ consists of at most finitely many $A_{1}$-singularities. Let $\hat{X} \rightarrow X$ be the blow up $X$ in $\Sigma$ and let $Q$ be the union of the exceptional quadrics. Consider the natural maps $\alpha: H^{2}(\hat{X}) \rightarrow H^{2}(H)$ and $\beta: H^{2}(\hat{X}) \rightarrow H^{2}(Q)$. Then $\beta$ is injective on the kernel of $\alpha$.

Proof. Note that $H^{2}(H \cup Q)=H^{2}(H) \oplus H^{2}(Q)$ since $H \cap Q=\varnothing$. It follows that $H^{2}(H \cup Q, H) \cong H^{2}(Q)$ and we have a commutative diagram

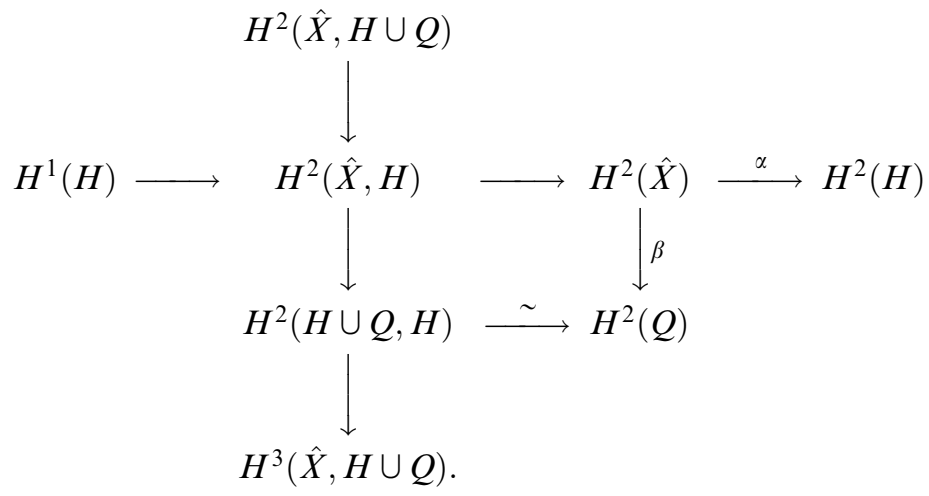

In order to prove the claim it is sufficient to show that $H^{2}(\hat{X}, H \cup Q)=0$. By Alexander duality

$$
H^{2}(\hat{X}, H \cup Q)=H_{4}(\hat{X} \backslash(H \cup Q)) \cong H_{4}(W \backslash \Sigma),
$$

where $W:=X \backslash H$. Since $W$ is affine of dimension 3 we know that $H_{k}(W)=0$ for $k \geqslant 4$. The exact homology sequence for the pair $(W, W \backslash \Sigma)$ gives

$$
H_{5}(W, W \backslash \Sigma) \rightarrow H_{4}(W \backslash \Sigma) \rightarrow H_{4}(W) .
$$


Since $H_{4}(W)=0$ it is enough to prove that $H_{5}(W, W \backslash \Sigma)=0$. Let us first assume that $\Sigma=\{p\}$. Then by an excision argument $H_{5}(W, W \backslash \Sigma) \cong H_{5}(U, U \backslash p)$ where $U$ is a suitable contractible neighbourhood of the point $p$. It remains to show that $H_{5}(U, U \backslash p)=0$. This is a local problem. Let $Q^{\prime} \subset \mathbb{C}^{4}$ be the quadric cone and let $K=S_{\varepsilon} \cap Q^{\prime}$ be the link. Since $U$ is contractible, we find $H_{5}(U, U \backslash p) \cong H_{4}(K)$. The manifold $K$ is orientable and 1-connected (see [6, p. 76]). Hence $H_{4}(K) \cong H_{1}(K) \cong 0$ where the first isomorphism is by Poincaré duality. The general case where $\Sigma$ consists of several points can be done by induction by removing one point at a time.

Remark 2.18. Note that the proof works for arbitrary isolated hypersurface singularities.

As mentioned before, we will apply the lemma to $X=\tilde{N}^{(3)}$. For $H$ we will take the pull back of

$$
h:=E_{1}^{(3)}+E_{2}^{(3)}+E_{3} .
$$

Lemma 2.19. The pair $\left(\tilde{N}^{(3)}, H\right)$ satisfies the conditions of Lemma 2.17.

Proof. It suffices to show that $a_{0} \sum S_{k}^{(3)}+a_{1} E_{1}^{(3)}+a_{2} E_{2}^{(3)}+a_{3} E_{3}$ is very ample on $\mathbb{P}^{(3)}$ for suitable integers $a_{0}, a_{1}, a_{2}, a_{3}>0$. Indeed, its restriction to $N^{(3)}$ has the same support as $h$, since $S_{k}^{(3)} \cap N^{(3)}=\varnothing$. Hence its pullback $H$ supports an ample divisor on $\tilde{N}^{(3)}$, since $\tilde{N}^{(3)} \rightarrow \tilde{N}$ is finite. This implies that the complement $\tilde{N}^{(3)} \backslash H$ is affine. We also know that $\tilde{N}^{(3)} \backslash H$ is non-singular outside the Segre double points.

It remains to prove the claim about $a_{0} \sum S_{k}^{(3)}+a_{1} E_{1}^{(3)}+a_{2} E_{2}^{(3)}+a_{3} E_{3}$. Let $H_{0}:=$ $\sum S_{k}$ in $\mathbb{P}^{(0)}$. The divisor $H_{1}:=p_{1}^{*}\left(n_{1} H_{0}\right)-E_{1}$ is very ample on $\mathbb{P}^{(1)}$ for $n_{1} \gg 0$. In fact it suffices that $\mathscr{I}_{\mathscr{P}}\left(6 n_{1}-1\right)$ be globally generated, where $\mathscr{P}=\left\{P_{k \ell m n}\right\}$ (see [12, proof of Proposition II.7.10 (b)]). Similarly, $H_{2}:=p_{2}^{*}\left(n_{2} H_{1}\right)-E_{2}$ is very ample on $\mathbb{P}^{(2)}$ for $n_{2} \gg n_{1} \gg 0$ and $H_{3}:=p_{3}^{*}\left(n_{3} H_{2}\right)-E_{3}$ is very ample on $\mathbb{P}^{(3)}$ for $n_{3} \gg n_{2} \gg$ $n_{1} \gg 0$. Using $p_{1}^{*}\left(\sum S_{k}\right)=\sum S_{k}^{(1)}+4 E_{1}$, one finds that $H_{1}=n_{1} \sum S_{k}^{(1)}+\left(4 n_{1}-1\right)$. $E_{1}$. Continuing in this fashion for $H_{2}$ and $H_{3}$, one finds $H_{3}=a_{0} \sum S_{k}^{(3)}+a_{1} E_{1}^{(3)}+$ $a_{2} E_{2}^{(3)}+a_{3} E_{3}$ with $a_{0}=n_{3} n_{2} n_{1}, a_{1}=n_{3} n_{2}\left(4 n_{1}-1\right), a_{2}=n_{3}\left(3 n_{2} n_{1}-1\right)$ and $a_{3}=$ $2 n_{3} n_{2} n_{1}-1$. So $H_{3}$ is indeed very ample for suitable $a_{0}, a_{1}, a_{2}, a_{3}>0$.

Lemma 2.20. Let $Q$ be an exceptional quadric obtained by blowing up $\tilde{N}^{(3)}$ in a Segre node. Then condition $(* *)$ holds for $Q$.

Proof. We may assume that the node is $(1: 1: 1:-1:-1:-1)$. The tangent cone, which is a cone over $Q$, is given by the equation

$$
x_{0}+\cdots+x_{5}=x_{0}^{2}+x_{1}^{2}+x_{2}^{2}-x_{3}^{2}-x_{4}^{2}-x_{5}^{2}=0
$$

in $\mathbb{P}_{5}$ (see $\left[1\right.$, p. 181]). So the rulings of $Q$ are defined over $\mathbb{F}_{p}$. 
Proposition 2.21. Condition (**) holds for $\tilde{N}^{(3)}$.

Proof. Let $X=\tilde{N}^{(3)}$ and let $\hat{X}$ be the blow up of $X$ in the 20 Segre nodes. The Leray spectral sequence of $\hat{X} \rightarrow X$ induces an injection $H^{2}(X) \hookrightarrow H^{2}(\hat{X})$, so it suffices to prove condition $(* *)$ for $\hat{X}$. By Lemma 2.17, the comparison theorem between étale and complex cohomology, and the lemma above it would even suffice to check condition $(* *)$ for $H$. However, we will see in a moment that condition $(* *)$ probably does not hold for $H$. This is not a serious problem. Indeed, we just restrict our attention to eigenvalues of absolute value $p$ in the conditions $(*)_{p},(*)$ and $(* *)$. Call the (weaker) modified conditions $(*)_{p}^{\prime},(*)^{\prime}$ and $(* *)^{\prime}$, respectively. For smooth proper varieties the modified conditions are equivalent to the original ones. Furthermore, the modified conditions are again stable under extensions of Galois modules. So it suffices to prove that $(* *)^{\prime}$ holds for $H$.

Note that $h=E_{1}^{(3)} \cup E_{2}^{(3)} \cup E_{3}$ is a divisor with global normal crossings, i.e. its components are smooth and meet each other transversally. The same holds for the pull back $H$. So we can apply the Mayer-Vietoris spectral sequence (cf. [15, p. 103]). This spectral sequence abuts to $H^{p+q}(H)$, degenerates at $E_{2}$ and has $E_{1}^{p, q}=$ $H^{q}(X[p])$, where $H[i]$ is the disjoint union of strata of codimension $i$, i.e. $H[0]$ consists of the triple points, $H[1]$ is the disjoint union of the intersection curves and $H[0]$ is the disjoint union of the smooth components of $H$. Now note that the components of $H[1]$ are rational curves, hence $E_{1}^{1,1}=0$. So in our case we get an exact sequence

$$
H^{0}(H[1]) \stackrel{d_{1}^{1,0}}{\longrightarrow} H^{0}(H[2]) \longrightarrow H^{2}(H) \longrightarrow H^{2}(H[0]) \stackrel{d_{1}^{0,2}}{\longrightarrow} H^{2}(H[1]) .
$$

This exact sequence is related to the weight filtration on $H^{2}(H)$ : one has $W_{0}\left(H^{2}(H)\right)$ $=W_{1}\left(H^{2}(H)\right)=E_{\infty}^{2,0}=\operatorname{Coker}\left(d_{1}^{1,0}\right) \quad$ and $\operatorname{Gr}_{2}^{W}\left(H^{2}(H)\right)=E_{\infty}^{0,2}=\operatorname{Ker}\left(d_{1}^{0,2}\right) \quad(\mathrm{cf}$. [15]). Corollary 2.9 implies that condition (**) holds for $\mathrm{Gr}_{2}^{W} \subseteq H^{2}(H[0])$. On the other hand, Frob ${ }_{p}=$ id on $W_{0}$ (cf. $\left.[5, \S 14]\right)$, so condition $(* *)$ does not hold on $W_{0}$ if $W_{0} \neq 0$ ! However, as we remarked in the first paragraph of this proof, it suffices to note that the weaker condition $(* *)^{\prime}$ clearly holds for $W_{0}$.

Remark 2.22. Alternatively, one can use the strictness of the weight filtration (cf. [5, $\S 1])$. Indeed, we want to show that for $p \equiv 1 \bmod 4$ the eigenvalues of $\mathrm{Frob}_{p}$ on the image $I$ of $\alpha: H^{2}(\hat{X}) \rightarrow H^{2}(H)$ are all equal to $p$. Strictness of the weight filtration implies that $I \cap W_{1}=0$, i.e. that $I$ injects into $\mathrm{Gr}_{2}^{W}\left(H^{2}(H)\right) \subseteq H^{2}(H[0])$, where we know condition $(* *)$ by Corollary 2.9 .

\section{The $L$-function of $Y$ and $\tilde{Y}$}

In this section we will show that the $L$-functions of $H_{\text {èt }}^{3}(Y)$ and $H_{\text {ét }}^{3}(\tilde{Y})$ are modular. In fact, the $L$-functions are equal and belong to the modular form 


$$
\begin{aligned}
f(q):= & \left(\eta(q) \eta\left(q^{2}\right) \eta\left(q^{3}\right) \eta\left(q^{6}\right)\right)^{2} \\
= & q \prod_{n=1}^{\infty}\left(1-q^{n}\right)^{2}\left(1-q^{2 n}\right)^{2}\left(1-q^{3 n}\right)^{2}\left(1-q^{6 n}\right)^{2} \\
= & q-2 q^{2}-3 q^{3}+4 q^{4}+6 q^{5}+6 q^{6}-16 q^{7} \\
& -8 q^{8}+9 q^{9}-12 q^{10}+12 q^{11}-12 q^{12}+38 q^{13}+\cdots \\
= & : \sum_{n=1}^{\infty} a_{n} q^{n} .
\end{aligned}
$$

$f$ is a cusp form of weight 4 with respect to the groups

$$
\Gamma_{0}(6):=\left\{\left(\begin{array}{ll}
a & b \\
c & d
\end{array}\right) \in \mathrm{SL}_{2}(\mathbb{Z}): c \equiv 0 \bmod 6\right\}
$$

and

$$
\Gamma_{1}(6):=\left\{\left(\begin{array}{ll}
a & b \\
c & d
\end{array}\right) \in \Gamma_{0}(6): a \equiv 1 \bmod 6\right\}
$$

These groups define the same modular forms of even weight since their images in $\mathrm{PSL}_{2}(\mathbb{Z})$ coincide. For the same reason, the natural map $X_{1}(6) \rightarrow X_{0}(6)$ between the associated modular curves is an isomorphism. We denote the universal elliptic curve over $X_{1}(6)$ by $S_{1}(6)$. Standard formulae imply that the space $S_{4}\left(\Gamma_{0}(6)\right)$ of cusp forms of weight 4 has dimension 1 and it is well known that $f$ generates this space [18]. Furthermore, it is a (normalized) newform, since there are no cusp forms of weight 4 and level 2 or 3.

The $L$-function of $f$ is the Mellin transform

$$
L(f, s):=\sum_{n=1}^{\infty} a_{n} n^{-s}
$$

of $f$. Since $a_{n}=O\left(n^{3 / 2}\right)$ for $n \rightarrow \infty$, it converges for $\Re(s)>5 / 2$. It has an analytic continuation to an entire function. Furthermore, there is a functional equation relating $L(f, s)$ and $L(f, 4-s)$. Since $f$ is a Hecke eigenform, its $L$-function is an Euler product

$$
L(f, s)=\prod_{p \text { prime }} L_{p}(f, s)
$$

with Euler factors

$$
L_{p}(f, s)=\frac{1}{1-a_{p} p^{-s}+p^{3} \cdot p^{-2 s}} \quad \text { for } p \geqslant 5
$$

and $L_{p}(f, s)=\left(1+p \cdot p^{-s}\right)^{-1}$ for $p<5$. 
The $L$-function of the Galois module $H_{\text {èt }}^{3}(Y)$ is also an Euler product. Its Euler factor at a prime $p \geqslant 5$ is

$$
L_{p}\left(H_{\mathrm{et}}^{3}(Y), s\right)=\frac{1}{1-a_{p}(Y) p^{-s}+p^{3} \cdot p^{-2 s}},
$$

where $a_{p}(Y):=t_{3}:=\operatorname{tr}\left(\operatorname{Frob}_{p}^{*} ; H_{\text {ét }}^{3}(Y)\right)$. The $L$-function of $H_{\text {êt }}^{3}(\tilde{Y})$ is defined analogously.

Our proof of the modularity of $L\left(H_{\text {ét }}^{3}(Y), s\right)$ will follow the lines of [21, §3.5]. The main point is that a theorem due to Serre (see also [17]), based on Faltings work and recast by Livné, essentially reduces the proof to checking equality of the Fourier coefficients for only finitely many primes! To make this precise we recall Livné's theorem [14, Theorem 4.3].

Theorem 3.1. Let $S$ be a finite set of prime numbers and let $\rho_{1}, \rho_{2}$ be continuous 2-dimensional 2-adic representations of $\operatorname{Gal}(\overline{\mathbb{Q}} / \mathbb{Q})$ unramified outside $S$. Let $\mathbb{Q}_{S}$ be the compositum of all quadratic extensions of $\mathbb{Q}$ which are unramified outside $S$. Let $T$ be a set of primes, disjoint from $S$, such that $\operatorname{Gal}\left(\mathbb{Q}_{S} / \mathbb{Q}\right)=\left\{\left.\operatorname{Frob}_{p}\right|_{\mathbb{Q}_{S}}: p \in T\right\}$. Suppose that

(a) $\operatorname{tr} \rho_{1}\left(\right.$ Frob $\left._{p}\right)=\operatorname{tr} \rho_{2}\left(\right.$ Frob $\left._{p}\right)$ for all $p \in T$;

(b) $\operatorname{det} \rho_{1}\left(\right.$ Frob $\left._{p}\right)=\operatorname{det} \rho_{2}\left(\right.$ Frob $\left._{p}\right)$ for all $p \in T$;

(c) $\operatorname{tr} \rho_{1} \equiv \operatorname{tr} \rho_{2} \equiv 0 \bmod 2$ and $\operatorname{det} \rho_{1} \equiv \operatorname{det} \rho_{2} \bmod 2$.

Then $\rho_{1}$ and $\rho_{2}$ have isomorphic semisimplifications, whence $L\left(\rho_{1}, s\right)=L\left(\rho_{2}, s\right)$. In particular, the good Euler factors of $\rho_{1}$ and $\rho_{2}$ coincide.

Theorem 3.2. We have $L\left(H_{\text {ét }}^{3}(Y), s\right) \stackrel{\circ}{=}\left(H_{\text {êt }}^{3}(\tilde{Y}), s\right) \stackrel{\circ}{=} L(f, s)$, i.e. the Euler factors for $p \geqslant 5$ coincide.

Proof. Note that $L\left(H_{\text {ét }}^{3}(\tilde{Y}), s\right) \stackrel{\circ}{=} L\left(H_{\text {èt }}^{3}(Y), s\right)$, since $\tilde{Y} \rightarrow Y$ is a finite map inducing an isomorphism on $H^{3,0}$. So it suffices to show that $L\left(H_{\text {ét }}^{3}(Y), s\right) \stackrel{\circ}{=} L(f, s)$.

We work with 2-adic cohomology (because of Livnés theorem). Recall that $L(f, s)=L\left(\rho_{f}, s\right)$, where $\rho_{f}: \operatorname{Gal}(\overline{\mathbb{Q}} / \mathbb{Q}) \rightarrow \operatorname{Aut}(F)$ is the 2-dimensional 2-adic representation associated to $f$ by Deligne [4]. The $L$-series $L\left(H_{\text {ett }}^{3}(Y), s\right)$ also depends on the Galois module structure of $H_{\text {ét }}^{3}(Y)$, so $L\left(\rho_{Y}, s\right)$ would be a more appropriate notation, where $\rho_{Y}$ denotes the action of $\operatorname{Gal}(\overline{\mathbb{Q}} / \mathbb{Q})$ on $H_{\text {ét }}^{3}(Y)$. We apply Livné's theorem to $\rho_{1}=\rho_{Y}, \rho_{2}=\rho_{f}$ and $S=\{2,3\}$. We can take $T=\{5,7,11,13,17,19$, $23,73\}$, since $\mathbb{Q}_{S}=\mathbb{Q}(\sqrt{-1}, \sqrt{2}, \sqrt{3})=\mathbb{Q}\left(e^{2 \pi i / 24}\right)$ and the image of Frob $\left._{p}\right|_{\mathbb{Q}_{S}}$ under $\operatorname{Gal}\left(\mathbb{Q}_{S} / \mathbb{Q}\right) \stackrel{\sim}{\rightarrow}(\mathbb{Z} / 24 \mathbb{Z})^{*}$ is simply $p \bmod 24$.

We claim that the conditions (a), (b) and (c) of Livné's theorem follow from

(i) the $p$-th Fourier coefficient of $f$ coincides with the trace $t_{3}(p)$ of Frob $_{p}$ acting on $\left.H_{\text {èt }}^{3}(Y)\right)$ for $p \in\{5,7,11,13,17,19,23,73\}=T$; 
(ii) $\operatorname{det}\left(\operatorname{Frob}_{p} ; H_{\text {êt }}^{3}(Y)\right)=+p^{3}$ for all $p \in T$;

(iii) $t_{3}(p)$ is even for all $p \geqslant 5$.

Indeed, Livné's conditions (a) and (b) specialize to (i) and (ii) respectively, since $\operatorname{det}\left(\rho_{f}\left(\operatorname{Frob}_{p}\right)\right)=p^{3}$ by Deligne or by inspection of the $p$-th Euler factor of $L(f, s)$. By Chebotarev's density theorem it suffices to check condition $(c)$ in Frob for almost all $p$. Since for $p \geqslant 5$ each determinant equals $\pm p^{3}$, we have $\operatorname{det} \rho_{Y}\left(\operatorname{Frob}_{p}\right) \equiv 1 \equiv$ $\operatorname{det} \rho_{f}\left(\operatorname{Frob}_{p}\right) \bmod 2$. Finally, the evenness of $\operatorname{tr} \rho_{f}\left(\operatorname{Frob}_{p}\right)$ for $p \geqslant 5$ was proved [21, Lemma 3.12], so condition (c) indeed reduces to condition (iii).

It remains to prove the conditions (i), (ii) and (iii). Recall that

$$
\begin{aligned}
t_{3} & =\left(1+t_{2}+t_{4}+p^{3}\right)-\# Y\left(\mathbb{F}_{p}\right) \\
& =\left(1+50 p+50 p^{2}+p^{3}\right)-\# Y\left(\mathbb{F}_{p}\right)
\end{aligned}
$$

(cf. Theorem 2.1 and Proposition 2.4). By the formula $\# Y\left(\mathbb{F}_{p}\right)=\# U\left(\mathbb{F}_{p}\right)+50 p^{2}+$ $50 p+20$ this boils down to

$$
t_{3}=p^{3}-19-\# U\left(\mathbb{F}_{p}\right) .
$$

Using a computer to determine $\# U\left(\mathbb{F}_{p}\right)$ we get the following table.

\begin{tabular}{r|r|r|r}
$p$ & $\# U\left(\mathbb{F}_{p}\right)$ & $\# Y\left(\mathbb{F}_{p}\right)$ & \multicolumn{1}{|c}{$t_{3}$} \\
\hline 5 & 100 & 1620 & 6 \\
7 & 340 & 3160 & -16 \\
11 & 1300 & 7920 & 12 \\
13 & 2140 & 11260 & 38 \\
17 & 5020 & 20340 & -126 \\
19 & 6820 & 25840 & 20 \\
23 & 11980 & 39600 & 168 \\
73 & 388780 & 658900 & 218
\end{tabular}

We wrote a straightforward Maple program to compute these numbers. The computation of $\# U\left(\mathbb{F}_{23}\right)$ took less than a minute on a Macintosh $\mathrm{G} 4$ with a $350 \mathrm{MHz}$ processor; the computation of $\# U\left(\mathbb{F}_{73}\right)$ took about 80 minutes. Using more advanced techniques one can speed up these calculations considerably. Note that $t_{3}$ indeed coincides with the corresponding Fourier coefficient of $f$ for these primes. This proves condition (i).

Condition (ii) follows from the observation that $t_{3} \neq 0$ for the 8 primes above. Indeed, if $\alpha$ and $\beta$ denote the eigenvalues of Frobenius on $H_{\mathrm{et}}^{3}$, then $\operatorname{det}\left(\operatorname{Frob}_{p}\right)=\alpha \beta$ is an integer of absolute value $p^{3}$ and we only have to exclude the case $\{\alpha, \beta\}=$ $\left\{-p^{3 / 2}, p^{3 / 2}\right\}$. However, in that case we would have $t_{3}=\alpha+\beta=0$.

Finally, we have to check that $\# U\left(\mathbb{F}_{p}\right)$ is even for every prime $p \geqslant 5$. Consider the involution $\left(x_{0}: \cdots: x_{5}\right) \mapsto\left(x_{0}^{-1}: \cdots: x_{5}^{-1}\right)$ on $U=\left\{x \in N: x_{0} \ldots x_{5} \neq 0\right\}$. Its fixpoints are the 10 Segre points. Since the number of fixpoints is even, so is $\# U\left(\mathbb{F}_{p}\right)$. 
Remark 3.3. This implies that $a_{p}=\left(1+60 p+60 p^{2}+p^{3}\right)-\# \tilde{Y}\left(\mathbb{F}_{p}\right)$ for $p \equiv 1 \bmod$ 4 , since $t_{2}:=\operatorname{tr}\left(\operatorname{Frob}_{p} ; H_{\text {ét }}^{2}(\tilde{Y})\right)=60 p$ and $t_{4}:=\operatorname{tr}\left(\operatorname{Frob}_{p} ; H_{\text {èt }}^{4}(\tilde{Y})\right)=60 p^{2}$ in this case. Conversely, one can use Theorem 3.2 to determine $t_{2}$ and $t_{4}$ on $\tilde{Y}$ for $p \equiv 3 \bmod 4$. Indeed, our proof of condition $(* *)$ also shows that the eigenvalues of $\mathrm{Frob}_{p}$ on $H_{\text {ét }}^{2}(\tilde{Y})$ are $\pm p$ for $p \equiv 3 \bmod 4$. Hence $\left(t_{2}, t_{4}\right)=k\left(p, p^{2}\right)$ for some integer $k$. The inequality

$$
\left|\left(1+k p+k p^{2}+p^{3}\right)-\# \tilde{Y}\left(\mathbb{F}_{p}\right)\right|<2 p^{3 / 2}
$$

and a computer computation of $\# \tilde{Y}\left(\mathbb{F}_{p}\right)$ then shows that $k=40$ for $p=7,11$, $19, \ldots, 59$. (In particular, for these primes the variety $\tilde{Y}$ does not satisfy condition $\left.(*)_{p}.\right)$ Of course, we expect that $k=40$ for all primes $p$ satisfying $p \equiv 3 \bmod 4, p \neq 3$, but we did not try to prove this.

\section{Correspondences with relatives}

We proved in Section 3 that

$$
L\left(H_{\text {èt }}^{3}(Y), s\right) \stackrel{\circ}{=}\left(H_{\text {èt }}^{3}(\tilde{Y}, s) \stackrel{\circ}{=} L(f, s)\right.
$$

where $f(q)=\left(\eta(q) \eta\left(q^{2}\right) \eta\left(q^{3}\right) \eta\left(q^{6}\right)\right)^{2}$ is the normalized generator of $S_{4}\left(\Gamma_{0}(6)\right)=$ $S_{4}\left(\Gamma_{1}(6)\right)$. The Tate conjecture then says that there should be a correspondence between $Y$ and $W=S_{1}(6) \times_{X_{1}(6)} S_{1}(6)$. We shall in fact see that there is a birational equivalence between $Y$ and $W$ which is defined over $\mathbb{Q}$.

Recall from [3] that the universal elliptic curve $S_{1}(6) \rightarrow X_{1}(6) \cong \mathbb{P}_{1}$ is given by the pencil

$$
(X+Y)(Y+Z)(Z+X)+t X Y Z=0
$$

Theorem 4.1. There exists a birational equivalence between $S_{1}(6) \times_{X_{1}(6)} S_{1}(6)$ and $Y$ which is defined over $\mathbb{Q}$.

Proof. One finds immediately from the defining equations of the Barth-Nieto quintic that

$$
x_{0}+x_{1}+x_{2}=-\left(x_{3}+x_{4}+x_{5}\right), \quad \frac{1}{x_{0}}+\frac{1}{x_{1}}+\frac{1}{x_{2}}=-\left(\frac{1}{x_{3}}+\frac{1}{x_{4}}+\frac{1}{x_{5}}\right) .
$$

By multiplying these two equations we obtain two pencils of cubics, namely

$$
\left(x_{0}+x_{1}+x_{2}\right)\left(\frac{1}{x_{0}}+\frac{1}{x_{1}}+\frac{1}{x_{2}}\right)=\left(x_{3}+x_{4}+x_{5}\right)\left(\frac{1}{x_{3}}+\frac{1}{x_{4}}+\frac{1}{x_{5}}\right)=: t^{\prime} .
$$


The first (and similarly the second) pencil can also be written in the form

$$
\left(x_{0}+x_{1}+x_{2}\right)\left(x_{1} x_{2}+x_{2} x_{0}+x_{0} x_{1}\right)=t^{\prime} x_{0} x_{1} x_{2}
$$

or equivalently

$$
\left(x_{0}+x_{1}\right)\left(x_{1}+x_{2}\right)\left(x_{2}+x_{0}\right)+\left(1-t^{\prime}\right) x_{0} x_{1} x_{2}=0 .
$$

Setting $t=1-t^{\prime}$ this is precisely Beauville's pencil. Hence the rational map given by

$$
\left(x_{0}: \cdots: x_{5}\right) \mapsto\left(\left(x_{0}: x_{1}: x_{2}\right), t,\left(x_{3}: x_{4}: x_{5}\right)\right)
$$

defines a rational equivalence between $Y \subset \mathbb{P}_{5}$ and $W \subset \mathbb{P}_{2} \times \mathbb{P}_{1} \times \mathbb{P}_{2}$.

Remark 4.2. The analogous procedure using the equations

$$
x_{0}+x_{1}=-\left(x_{2}+x_{3}+x_{4}+x_{5}\right) \text { and } \quad \frac{1}{x_{0}}+\frac{1}{x_{1}}=-\left(\frac{1}{x_{2}}+\frac{1}{x_{3}}+\frac{1}{x_{4}}+\frac{1}{x_{5}}\right)
$$

gives rise to a pencil of $\mathrm{K} 3$-surfaces on $Y$.

H. Verrill has studied in [21] a Calabi-Yau variety $\mathscr{Z}_{A_{3}}$ which is the smooth model of the variety $V$ given in inhomogeneous coordinates by

$$
V:(1+x+x y+x y z)(1+z+y z+x y z)=\frac{(t+1)^{2}}{t} x y z .
$$

She has shown that $L\left(H_{\text {ét }}^{3}\left(\mathscr{Z}_{A_{3}}\right), s\right) \stackrel{\circ}{=}(f, s)$ and hence one also expects a correspondence between $Y$ and $\mathscr{Z}_{A_{3}}$, resp. $N$ and $V$.

Theorem 4.3. There exists a birational equivalence between $N$ and $V$ which is defined over $\mathbb{Q}$.

Proof. The variety $V$ is fibred by a pencil of K3-surfaces. In order to find a suitable fibration of $Y$ we consider the pencil $H_{t}=\left\{x_{0}=t x_{1}\right\}$ containing the plane $F_{01}=$ $\left\{x_{0}=x_{1}=0\right\}$ and a residual quartic surface $X_{t}$, i.e.

$$
H_{t} \cap N=F_{01} \cup X_{t} .
$$

Combining the equations $x_{0}=t x_{1}$ and $x_{0}+\cdots+x_{5}=0$ we obtain

$$
x_{1}=-\frac{1}{t+1}\left(x_{2}+x_{3}+x_{4}+x_{5}\right) \quad \text { and } \quad x_{0}=-\frac{t}{t+1}\left(x_{2}+x_{3}+x_{4}+x_{5}\right) \text {. }
$$

Substituting this into the second defining equation of $N$ we get

$$
\sum_{i=0}^{5} x_{0} \ldots \hat{x}_{i} \ldots x_{5}=\left(x_{2}+x_{3}+x_{4}+x_{5}\right)\left(\frac{t}{(t+1)^{2}} e_{1} e_{3}-e_{4}\right)
$$


where $e_{j}=e_{j}\left(x_{2}, x_{3}, x_{4}, x_{5}\right)$ is the $j$-th elementary symmetric function. Hence the residual quartic $X_{t}$ is given by

$$
X_{t}: e_{1} e_{3}-\frac{(t+1)^{2}}{t} e_{4}=0
$$

We consider the Cremona transformation of $\mathbb{P}_{3}$ given by

$$
\left(x_{2}: x_{3}: x_{4}: x_{5}\right)=(1: x: x y: x y z)
$$

Note that under this transformation

$$
\begin{aligned}
& e_{1}=1+x+x y+x y z \\
& e_{3}=x^{2} y \cdot(1+z+y z+x y z) \\
& e_{4}=x^{2} y \cdot x y z
\end{aligned}
$$

Comparing this with the equations defining $V$ shows that the rational map $\left(x_{2}: x_{3}: x_{4}: x_{5}\right) \mapsto(1: x: x y: x y z): N \rightarrow V$ gives the desired birational equivalence.

Remark 4.4. 1. Combining the maps from Theorems 4.1 and 4.3 one obtains a birational equivalence between $V$ and $W$. Saito and Yui have also found an explicit birational equivalence between these varieties (cf. [23, p. 542]). Their map, however, differs from ours.

2. By a result of Batyrev [2] the two smooth Calabi-Yau varieties $Y$ and $\mathscr{Z}_{A_{3}}$ must have the same Betti numbers and hence also the same Hodge numbers. This agrees with our computations. Note however, that we still need the calculation of Section 2 to compute the Hodge numbers of $Z$.

The birational equivalence between $N$ and $W$ means that one can associate to each general pair of points $(P, Q)$ on the same fibre $E$ of $S_{1}(6) \rightarrow X_{1}(6)$ an $H_{2,2}$-invariant Kummer surface, resp. two abelian surfaces with a $(1,3)$-polarization and a level-2 structure. This can be made explicit in the following sense. Let $(P, Q) \in W$. Then we can choose homogeneous coordinates $P=\left(x_{0}: x_{1}: x_{2}\right)$ and $Q=\left(x_{3}: x_{4}: x_{5}\right)$ such that $x_{0}+\cdots+x_{5}=0$. Then $x_{0}, \ldots, x_{5}$ define a quartic surface

$$
X=\left\{\sum_{i=0}^{5} x_{i} t_{i}=0\right\} \subset \mathbb{P}_{3}
$$

where the $t_{i}$ are the $H_{2,2}$-invariant quartic polynomials given in [1, p. 189]. The surface $X$ contains 2 sets of 16 disjoint lines and taking the branched cover along these sets of 16 lines we obtain after blowing down the $(-1)$-curves two abelian surfaces $A$ 
and $A^{\prime}$. The line bundle $\mathcal{O}_{X}(1)$ defines a $(2,6)$-polarization, and hence also a $(1,3)$ polarization on $A$ and $A^{\prime}$ and the action of the Heisenberg group $H_{2,2}$ gives a level-2

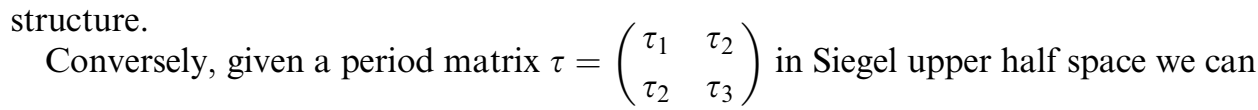
associate to it an abelian surface $A$ together with a $(1,3)$-polarization and a level-2 structure. These data define 4 explicitely known theta functions $\theta_{0}, \theta_{1}, \theta_{2}, \theta_{3}$ such that the map defined by these functions embed the Kummer surface $X$ of $A$ as an $H_{2,2}$ invariant quartic surface (see [13]). Using the derivatives of $\theta_{0}, \theta_{1}, \theta_{2}, \theta_{3}$ and Heisenberg symmetry one can, at least in principle, compute the 16 lines on $X$ in $\mathbb{P}_{3}$. There is then a unique $H_{2,2}$-invariant quartic of the form $\left\{\sum x_{i} t_{i}=0\right\}$ containing these lines and one obtains $X=\left\{\sum x_{i} t_{i}=0\right\}$. The pair $(P, Q) \in W$ with $P=\left(x_{0}: x_{1}: x_{2}\right)$ and $Q=\left(x_{3}: x_{4}: x_{5}\right)$ is then the element of $W$ associated to the point $[\tau] \in \mathscr{A}_{1,3}(2)$. The latter variety has $Z$ and $\tilde{Y}$ as smooth models.

Remark 4.5. Threefolds which have a two dimensional Galois representation in $H_{\text {ét }}^{3}$ which is known or conjectured to be associated to an elliptic modular form of weight 4 are not easy to find. Here we list, in order of the level $N$ of the corresponding modular form, the ones we are aware of. Note that in several cases correspondences between relatives are not (yet) known.

1. $N=6$. There is a unique newform, see Section 3. This family of threefolds contains the ones discussed in this paper, the Barth-Nieto quintic $N$, its double cover $\tilde{Y}$ which is birational to the moduli space $\mathscr{A}_{1,3}(2)$, Verrill's threefold $\mathscr{Z}_{A_{3}}$ and the fibred square $W$ of the universal universal curve over $\Gamma_{1}(6)$. These relatives are in correspondence with each other. Two other relatives were found in [20]: they are given by $\sigma_{1}=\alpha \sigma_{5}+\beta \sigma_{2} \sigma_{3}=0$ for $(\alpha: \beta)=(1: 1),(-2: 1)$, where the $\sigma_{i}$ are the elementary symmetric functions in 6 variables. No correspondences between any of these two and the other relatives are known. Note that the Barth-Nieto quintic corresponds to $(1: 0)$.

2. $N=8$. There is a unique newform in $S_{4}\left(\Gamma_{0}(8)\right)$. In this family we have the desingularization $X^{\prime}$ of a Siegel modular threefold, denoted by $X$ in [19, p. 56]. The variety $X$ is a complete intersection of four quadrics in $\mathbb{P}_{7}$

$$
\begin{aligned}
& Y_{0}^{2}=X_{0}^{2}+X_{1}^{2}+X_{2}^{2}+X_{3}^{2} \\
& Y_{1}^{2}=X_{0}^{2}+X_{1}^{2}-X_{2}^{2}-X_{3}^{2} \\
& Y_{2}^{2}=X_{0}^{2}-X_{1}^{2}+X_{2}^{2}-X_{3}^{2} \\
& Y_{3}^{2}=X_{0}^{2}-X_{1}^{2}-X_{2}^{2}+X_{3}^{2} .
\end{aligned}
$$

(There is a misprint in the equations in [19]; these equations agree with those given in the appendix of [22], where $X$ is denoted by $W$ and the variables $Y_{1}$ and $Y_{2}$ are interchanged.) A relative of $X$ is the fibred square $W$ of the universal universal 
curve over $\Gamma_{0}(8)$. Using the moduli interpretation of $X$ and $W$, a correspondence between them was found in [7]. Using the explicit equation for (an open part of) $W$

$$
U_{0}+U_{0}^{-1}+U_{1}+U_{1}^{-1}+U_{2}+U_{2}^{-1}+U_{3}+U_{3}^{-1}=0
$$

(cf. [19, p. 60]), J. Stienstra found a dominant rational map $X \rightarrow W$ given by

$$
U_{i}:=\left(Y_{i}+\sqrt{2} X_{i}\right) /\left(Y_{i}-\sqrt{2} X_{i}\right)
$$

(there are unfortunately also misprints in the formulae given in $[19$, p. 60$]$ for this map).

3. $N=9$. There is a unique newform in $S_{4}\left(\Gamma_{0}(9)\right)$ which is defined by a Hecke character of the field $\mathbb{Q}(\sqrt{-3})$. A threefold (a desingularization of the intersection of two cubics in $\mathbb{P}_{5}$ ) with this L-series is given in the appendix of [22]. Relatives are the fibred square of the universal universal curve over $\Gamma_{0}(9)$ and a product of 3 elliptic curves, defined over $\mathbb{Q}$, with $j$-invariant 0 . No correspondences between these relatives seem to be known.

4. $N=12$. There is a unique newform in $S_{4}\left(\Gamma_{0}(12)\right)$. In the appendix of [22] a complete intersection of a quadric and a quartic in $\mathbb{P}_{5}$ having this L-series is given.

5. $N=21$. The threefold defined by an equation with $(\alpha: \beta)=(-3: 1)$ as in the $N=6$ case has an L-series which corresponds to a newform in $S_{4}\left(\Gamma_{0}(21)\right)$, see [20].

6. $N=25$. There is a newform in $S_{4}\left(\Gamma_{0}(25)\right)$ characterized by the first 5 Fourier coefficients $\left(a_{1}, \ldots, a_{5}\right)=(1,1,7,-7,0)$. The Schoen quintic, the 3 -fold in $\mathbb{P}_{5}$ defined by

$$
X_{0}^{5}+X_{1}^{5}+X_{2}^{5}+X_{3}^{5}+X_{4}^{5}-5 X_{0} X_{1} X_{2} X_{3} X_{4} X_{5}=0
$$

has this L-series (see [17, Proposition 5.3]). A relative is indicated in Remark 5.6 of that paper (again a fibred square of a universal elliptic curve), but no correspondence is known.

7. $N=50$. The Hirzebruch quintic 3 -fold in $\mathbb{P}_{4}$, which has 126 nodes, has an $L$-function which corresponds to the newform in $S_{4}\left(\Gamma_{0}(50)\right)$ characterized by $\left(a_{1}, \ldots, a_{5}\right)=(1,2,-2,4,0)$ (see [22]). No relatives are (explicitly) known, but there is of course the usual fibred square of the universal curve.

Remark 4.6. Since $Z$ is birationally isomorphic to the Siegel modular threefold $\mathscr{A}_{1,3}(2)$, the holomorphic three form on $Z$ corresponds to a Siegel modular cusp form of weight 3 for the paramodular group $\Gamma_{1,3}(2) \subset \mathrm{Sp}(4, \mathbb{Q})$. In [11], this cusp form was identified as $\Delta_{1}^{3}$. One might expect that $\Delta_{1}^{3}$ is the Saito-Kurokawa lift of the elliptic modular form $f$, but we do not know whether this is indeed the case. In [19] (see also the previous remark for $N=8$ ) a similar situation occurred. 


\section{References}

[1] W. Barth, I. Nieto, Abelian surfaces of type $(1,3)$ and quartic surfaces with 16 skew lines. J. Algebraic Geom. 3 (1994), 173-222. Zbl 809.14027

[2] V. Batyrev, Birational Calabi-Yau $n$-folds have equal Betti numbers. In: New trends in Algebraic Geometry, Proceedings Warwick Euroconference 1996 (K. Hulek, F. Catanese, Ch. Peters, M. Reid, eds.), pp. 1-11, London Math. Soc. Lecture Note Ser. 264, Cambridge Univ. Press, 1999. Zbl 990.67874

[3] A. Beauville, Les familles stables de courbes elliptiques sur $\mathbb{P}^{1}$ admettant quatre fibres singulières. C. R. Acad. Sci. Paris Sér. I Math. 294 (1982), 657-660. Zbl 504.14016

[4] P. Deligne, Formes modulaires et représentations $\ell$-adiques. In: Sém. Bourbaki 1968/69, no. 355, Lecture Notes in Math. 179, pp. 139-172, Springer-Verlag, 1971. Zbl 206.49901

[5] P. Deligne, Poids dans la cohomologie des variétés algébriques. In: Actes du Congrès International des mathématiciens, pp. 79-84 Vancouver, 1974. Zbl 334.14011

[6] A. Dimca, Singularities and Topology of Hypersurfaces. Springer-Verlag, 1992. Zbl 753.57001

[7] T. Ekedahl, B. van Geemen, An exceptional isomorphism between modular varieties. In: Arithmetic algebraic geometry, Texel, 1989, pp. 51-74, Progr. Math. 89, Birkhäuser Boston, Boston, MA, 1991. Zbl 732.14006

[8] J.-M. Fontaine, B. Mazur, Geometric Galois representations. In: Elliptic Curves, Modular Forms and Fermat's Last Theorem, Hong Kong, 1993 (J. Coates, S. T. Yau, eds.), pp. 4178, International Press, 1995. Zbl 839.14011

[9] E. Freitag, R. Kiehl, Etale Cohomology and the Weil Conjecture. Ergeb. Math. Grenzgeb. (3) 3, Springer-Verlag, 1988. Zbl 643.14012

[10] V. Gritsenko, K. Hulek, Minimal Siegel modular threefolds. Math. Proc. Cambridge Philos. Soc. 123 (1998), 461-485. Zbl 930.11028

[11] V. Gritsenko, K. Hulek, The modular form of the Barth-Nieto quintic. Internat. Math. Res. Notices 17 (1999), 915-937. Zbl 943.11027

[12] R. Hartshorne, Algebraic Geometry. Graduate Texts in Math. 52, Springer-Verlag, 1977. Zbl 531.14001

[13] K. Hulek, I. Nieto, G. Sankaran, Degenerations of $(1,3)$ abelian surfaces and Kummer surfaces. In: Algebraic Geometry: Hirzebruch 70, Proceedings of the algebraic geometry conference in honor of F. Hirzebruch's 70th birthday, Stefan Banach International Mathematical Center, Warszawa, Poland, May 11-16, 1998 (P. Pragacz, M. Szurek, J. Wiśniewski, eds.), pp. 177-192, Contemp. Math. 241, Amer. Math. Soc., Providence, RI, 1999. Zbl 991.19226

[14] R. Livné, Cubic exponential sums and Galois representations. Current trends in arithmetical algebraic geometry, Proc. Summer Res. Conf., Arcata/Calif. 1985 (K. Ribet, ed.), pp. 247-261, Contemp. Math. 67, Amer. Math. Soc., Providence, RI, 1987. Zbl 621.14019

[15] D. Morrison, The Clemens-Schmid exact sequence and applications. In: Topics in transcendental algebraic geometry (Ph. Griffiths, ed.), pp. 101-119, Ann. of Math. Stud. 106, Princeton Univ. Press, 1984. Zbl 576.32034

[16] M.-H. Saito, N. Yui, The modularity conjecture for rigid Calabi-Yau threefolds over $\mathbb{Q}$. AG/0009041 (2000).

[17] Ch. Schoen, On the geometry of a special determinantal hypersurface associated to the Mumford-Horrocks vector bundle. J. Reine Angew. Math. 364 (1986), 85-111.

Zbl 568.14022 
[18] G. Shimura, Introduction to the arithmetic theory of automorphic functions. Iwanmi Shoten and Princeton Univ. Press, Princeton, 1971. Zbl 872.11023

[19] B. van Geemen, N. Nygaard, On the geometry and arithmetic of some Siegel modular threefolds. J. Number Theory 53 (1995), 45-87. Zbl 838.11047

[20] D. van Straten, A quintic hypersurface in $\mathbb{P}^{4}$ with 130 nodes. Topology 32 (1993), 857 864. Zbl 801.14015

[21] H. Verrill, The L-series of certain rigid Calabi-Yau threefolds. J. Number Theory 81 (2000), 509-542.

[22] J. Werner, B. van Geemen, New examples of threefolds with $c_{1}=0$. Math. Z. 203 (1990), 211-225. Zbl 694.14020

[23] N. Yui, The arithmetic of certain Calabi-Yau varieties over number fields. In: The Arithmetic and Geometry of Algebraic Cycles, Proceedings of the NATO Advanced Study Institute, Banff, Canada, June 7-19, 1998 (B. Gordon, J. Lewis, S. Müller-Stach, Sh. Saito, N. Yui, eds.), pp. 515-560, NATO ASI Series. Series C. Mathematical and Physical Sciences 548, Kluwer, Dordrecht, 2000. Zbl 991.78175

[24] N. Yui, Arithmetic of certain Calabi-Yau varieties and mirror symmetry. IAS/Park City Proceedings on Arithmetic Geometry, AMS (to appear).

Received 6 November, 2000

K. Hulek, J. Spandaw, Institut für Mathematik, Universität Hannover, Postfach 6009, D 30060 Hannover, Germany

E-mail: hulek@math.uni-hannover.de spandaw@math.uni-hannover.de

B. van Geemen, Dipartimento di Matematica, Università di Pavia, Via Ferrata 1, I-27100 Pavia, Italy

E-mail: geemen@dragon.ian.pv.cnr.it

D. van Straten, Fachbereich Mathematik, FB17, Johannes Gutenberg Universität, Staudingerweg 9, 55099 Mainz, Germany

E-mail: straten@mathematik.uni-mainz.de 